\title{
How Water in Aliphatic Solvents Directs the Interference of Chemical Reactivity in a Supramolecular System
}

Mathijs F.J. Mabesoone ${ }^{1}$, Gijs M. ter Huurne ${ }^{1}$, Anja R.A. Palmans ${ }^{1}$, E.W.Meijer ${ }^{1 *}$

${ }^{1}$ Institute for Complex Molecular Systems and the Laboratory of Macromolecular and Organic Chemistry, Eindhoven University of Technology, P.O. Box 513, 5600 MB Eindhoven, The Netherlands

*Corresponding author: e.w.meijer@tue.nl

\section{Supplementary Information}




\section{Experimental details}

All starting materials were obtained from commercial suppliers and used without prior purification. $\mathbf{Z n - 1}$ was synthesized according to a previously reported procedure. ${ }^{1} \mathrm{MCH}$ and $\mathrm{CHCl}_{3}$ used in the spectroscopic measurements were of spectroscopic grade and purchased from VWR and TCl respectively. ${ }^{1} \mathrm{H}$ and ${ }^{13} \mathrm{C}$ NMR spectra were collected on a Bruker Avance $3 \mathrm{HD}$ NanoBay spectrometer $\left({ }^{1} \mathrm{H}\right.$ NMR $400 \mathrm{MHz},{ }^{13} \mathrm{C} \mathrm{NMR}$ $100 \mathrm{MHz}$ ) and measured in $\mathrm{CDCl}_{3} .{ }^{1} \mathrm{H}$ and ${ }^{13} \mathrm{C}$ shifts are reported relative to the residual $\mathrm{CHCl}_{3}$ signals at $7.26 \mathrm{ppm}$ and $77.23 \mathrm{ppm}$, respectively. MALDI-ToF mass spectrometry was performed with a Bruker autoflex Speed. CD spectroscopy was performed on a Jasco J-815 CD spectrometer equipped with an MPTC-490 multicell holder. UV-Vis results were obtained with the JASCO J-815 CD spectrometer or a Jasco V-650 UV-Vis spectrometer. All measurements with the porphyrins were performed in screw-capped cuvettes (Hellma Analytics). UV-Vis measurements on the Michael reactions without porphyrins were performed in Teflon capped cuvettes without $\mathrm{N}_{2}$ flow in the UV-Vis spectrometer. SLS data were collected in homemade air-tight, Teflon sealed screw-capped tubes. Water concentrations were determined in triplicate using a Mettler-Toledo C30 Coulometric KF Titrator with CombiCoulomat Frit KF reagent. Average values of the triplo are reported. SLS experiments were performed on an ALV Compact Goniometer System (CGS-3) Multi-Detector (MD-4) that was equipped with an ALV-7004 Digital Multiple Tau Real Time correlator, using a wavelength of $532 \mathrm{~nm}$. Scattering angles between 35 and $150^{\circ}$ were probed in steps of $5^{\circ}$. All SLS measurements were performed at $20^{\circ} \mathrm{C}$. AFM measurements were performed with an Asylum Research MFP-3D system in non-contact tapping mode. The obtained micrographs were processed with Gwyddion 2.52.

Column chromatography was performed a Biotage Isolera Spektra One Flash Chromatography system using KP-Sil Silica Gel SNAP column cartridges. HPLC-MS was performed with a Thermo Finnigan LCQ Fleet ion trap mass spectrometer equipped with a Surveyor PDA detector and Surveyor autosampler. Before analysis, the samples were separated on a reverse phase $\mathrm{C} 18$ column with an acetonitrile/water gradient with $0.1 \%$ formic acid with a flow speed of $0.2 \mathrm{~mL} / \mathrm{min}$ using Shimadzu SCL-10A pumps.

Association constants were determined using the Matlab code provided by Thordarson. ${ }^{2}$ A 1:1 binding model was assumed.

Preparation dry solvents: Dry $\mathrm{MCH}$ and $\mathrm{CHCl}_{3}$ were prepared by storing ambient $\mathrm{MCH}$ and $\mathrm{CHCl}_{3}$ over $4 \AA$ molecular sieves in a glove box for a minimum of 18 hours.

Preparation wet solvents: Wet $\mathrm{MCH}$ was prepared by stirring ambient $\mathrm{MCH}$ over a layer of water overnight. The $\mathrm{MCH}$ used for the sample preparation was pipetted from the phase-separated mixture.

Sample preparation titrations: A known amount between 1 and $2 \mathrm{mg}$ of $\mathbf{Z n - 1}$ or $\mathbf{H}_{\mathbf{2}} \mathbf{- 1}$ was dissolved in 100 $\mu \mathrm{LHCl}_{3}$ and $900 \mu \mathrm{L} \mathrm{MCH}$ was added after the pink solid was dissolved. NPrMal, PhSH, MA and MePip stocks were prepared in $\mathrm{MCH}$ by dissolving a known amount of material in a known amount of solvent. These stocks were used to prepare samples of $10 \mu \mathrm{M} \mathrm{Zn-1}$ or $\mathbf{H}_{2}-\mathbf{1}$ and the desired concentration of the additives. The samples were kept in Teflon-sealed vials and equilibrated overnight before measurement. Cuvettes with a path length of $1 \mathrm{~mm}$ were used for the experiments with $\mathbf{Z n - 1}$ and $\mathbf{H}_{\mathbf{2}} \mathbf{- 1}$.

All dry samples were prepared and transferred to the screw-capped cuvettes in a nitrogen filled glove box.

The titrations of $\mathbf{Z n}-\mathbf{M e}$ were performed in $\mathrm{MCH}$ sequentially adding aliquots of a stock solution containing a known amount of the piperidine catalyst and $1 \mu \mathrm{M} \mathrm{Zn}$-Me to a $2.5 \mathrm{~mL} 1 \mu \mathrm{M}$ solution of $\mathrm{Zn}$-Me to achieve 
a desired host-guest ratio. Spectra were collected immediately. Cuvettes with a path length of $1 \mathrm{~cm}$ were used.

Sample preparation SLS experiments: Samples were prepared in a similar way as described for the titrations. In addition, all solvents and stock solutions, except the porphyrin stock solution, were filtered through a $100 \mathrm{~nm}$ PTFE syringe filter before preparing the samples. After preparation of the samples in the desired compositions, the samples were filtered through a Whatman Puradisc 13 PTFE syringe filter with a $5.0 \mu \mathrm{m}$ pore size. The first $1.5 \mathrm{~mL}$ of the filtrate was discarded, after which the remaining filtrate was collected in sample tubes. Samples were equilibrated overnight and measured in home-made Teflonsealed, screw-capped sample tubes. Dry samples were prepared and transferred to the sample tubes in a nitrogen filled glove box. 


\section{Synthesis Zn-Me}

This procedure was adapted from a previous report. ${ }^{3}$

Zn-1 (49.5 mg, 16 umole) and Mel (41 $\mu \mathrm{L}, 0.659$ mmole) were dissolved in THF (10 mL) under an argon atmosphere and cooled to $0{ }^{\circ} \mathrm{C}$. Then, sodium hydride $(30.3 \mathrm{mg}, 0.758 \mathrm{mmole})$ in mineral oil $(60 \%)$ was added slowly to the reaction mixture. The mixture was left to stir and heat up to room temperature for 24 hours, after which the reaction was quenched with water $(3 \mathrm{~mL})$. Subsequently, the product was extracted into $\mathrm{CHCl}_{3}(50 \mathrm{~mL})$, after which the organic layer was washed with water and brine. The combined organic layers were then dried over $\mathrm{MgSO}_{4}$, filtered and the solvent was evaporated to yield a purple solid. After column chromatography (SNAP KP-Sil $10 \mathrm{~g}, \mathrm{CHCl}_{3}-\mathrm{EtOAc}, 0-100 \%$ ), the product was obtained as a purple solid (35 mg, 69\%). All NMR and MALDI-ToF data was in accordance with the previously reported results. ${ }^{1}$

\section{Synthesis $\mathrm{H}_{2} 1$}

Zn-1 (51.9 mg, 17 umole) was dissolved in $20 \mathrm{~mL} \mathrm{CHCl} 3$ in a separatory funnel. The organic layer was washed with $10 \mathrm{~mL} 37 \%$ hydrochloric acid, upon which the solution turned green. The layers were separated and the aqueous layer was extracted with $\mathrm{CHCl}_{3}$ until no green color could be observed in the aqueous phase anymore. Then, the combined organic layers were washed with saturated aqueous $\mathrm{Na}_{2} \mathrm{CO}_{3}$ solution, upon which the solution turned red. The organic layers were separated, and the aqueous layer was extracted with $\mathrm{CHCl}_{3}$ until no color could be observed in that layer anymore. Subsequently, the combined organic layers were dried over $\mathrm{MgSO}_{4}$, filtered and evaporated to yield the product as a purple solid (48.9 mg, 96\%). ${ }^{1} \mathrm{H}$ NMR (400 MHz, $\mathrm{CDCl}_{3}$ ) $\delta: 8.86$ (s, 8H, $\beta$-pyrrolic protons), 8.33 (d, 8H, ArH), 8.28 $(\mathrm{d}, 8 \mathrm{H}, \mathrm{ArH}), 8.06(\mathrm{~s}, 4 \mathrm{H}, \mathrm{NH}), 7.11(\mathrm{~s}, 8 \mathrm{H}, \mathrm{ArH}), 4.12\left(\mathrm{~m}, 16 \mathrm{H}, \mathrm{OCH}_{2}\right), 4.02\left(\mathrm{~m}, 8 \mathrm{H}, \mathrm{OCH}_{2}\right), 1.98-1.12(\mathrm{~m}$, $\left.120 \mathrm{H}, \mathrm{CH}_{2} / \mathrm{CH}\right), 0.99$ (d, $\left.36 \mathrm{H}, \mathrm{CH}_{3}\right), 0.90\left(\mathrm{~d}, 72 \mathrm{H}, \mathrm{CH}_{3}\right),-2.76,(\mathrm{~s}, 2 \mathrm{H}, \mathrm{NH}) .{ }^{13} \mathrm{C} \mathrm{NMR}\left(100 \mathrm{MHz}, \mathrm{CDCl}_{3}\right) \delta$ : 165.74, 153.69, 145.75, 135.67, 135.05, 134,94, 133.75, 125.69, 119.51, 99.67, 72.09, 67.85, 39.64, 37.82, $37.64,36.69,30.13,30.00,28.24,25.00,22.96,22.87,22.85,19.86$. MALDI-ToF analysis: Mass calc. 2964.21, mass observed: $2965.18\left(\mathrm{M}+\mathrm{H}^{+}\right)$.

\section{Synthesis Michael adduct}

$\mathrm{N}$-propylmaleimide ( $400 \mathrm{mg}, 2.9 \mathrm{mmole}$ ), thiophenol ( $317 \mathrm{mg}, 2,9 \mathrm{mmole}$ ) and $\mathrm{Et}_{3} \mathrm{~N}$ (9 mg, $72 \mu \mathrm{mole}$ ) were dissolved in $20 \mathrm{~mL} \mathrm{MeOH}$. The reaction was stirred at room temperature for 4 hours, after which the solvent was evaporated. After purification by column chromatography (KPSil 25g), the product was obtained as a yellow oil with quantitative yield. ${ }^{1} \mathrm{H} \mathrm{NMR}\left(400 \mathrm{MHz}, \mathrm{CDCl}_{3}\right) \delta: 7.51(2 \mathrm{H}, \mathrm{M}, \mathrm{ArH}), 7.35,(3 \mathrm{H}$, $\mathrm{M}, \mathrm{ArH}$ ), 4.00 (dd, $1 \mathrm{H}, \mathrm{CH}_{2}$ ), 3.38 (t, $2 \mathrm{H}, \mathrm{NCH}_{2}$ ), 3.13 (dd, $\left.1 \mathrm{H}, \mathrm{CH}_{2}\right), 2.70$ (dd, $1 \mathrm{H}, \mathrm{CH}_{2}$ ), 1.53-1.40 (m, $2 \mathrm{H}$, $\left.\mathrm{CH}_{2}\right), 0.81\left(\mathrm{t}, 3 \mathrm{H}, \mathrm{CH}_{3}\right) .{ }^{13} \mathrm{C} \mathrm{NMR}\left(100 \mathrm{MHz}, \mathrm{CDCl}_{3}\right) \delta: 175.68,174.73,134.72,130.50,129.55,129.50,44.14$, 10.91, 36.25, 21.05, 11.37. LC-MS: Mass calc. 249.08, mass observed: $250.18\left(\mathrm{M}+\mathrm{H}^{+}\right)$. 
${ }^{1} \mathrm{H}$ and ${ }^{13} \mathrm{C}$ NMR of $\mathrm{H}_{2}-1$

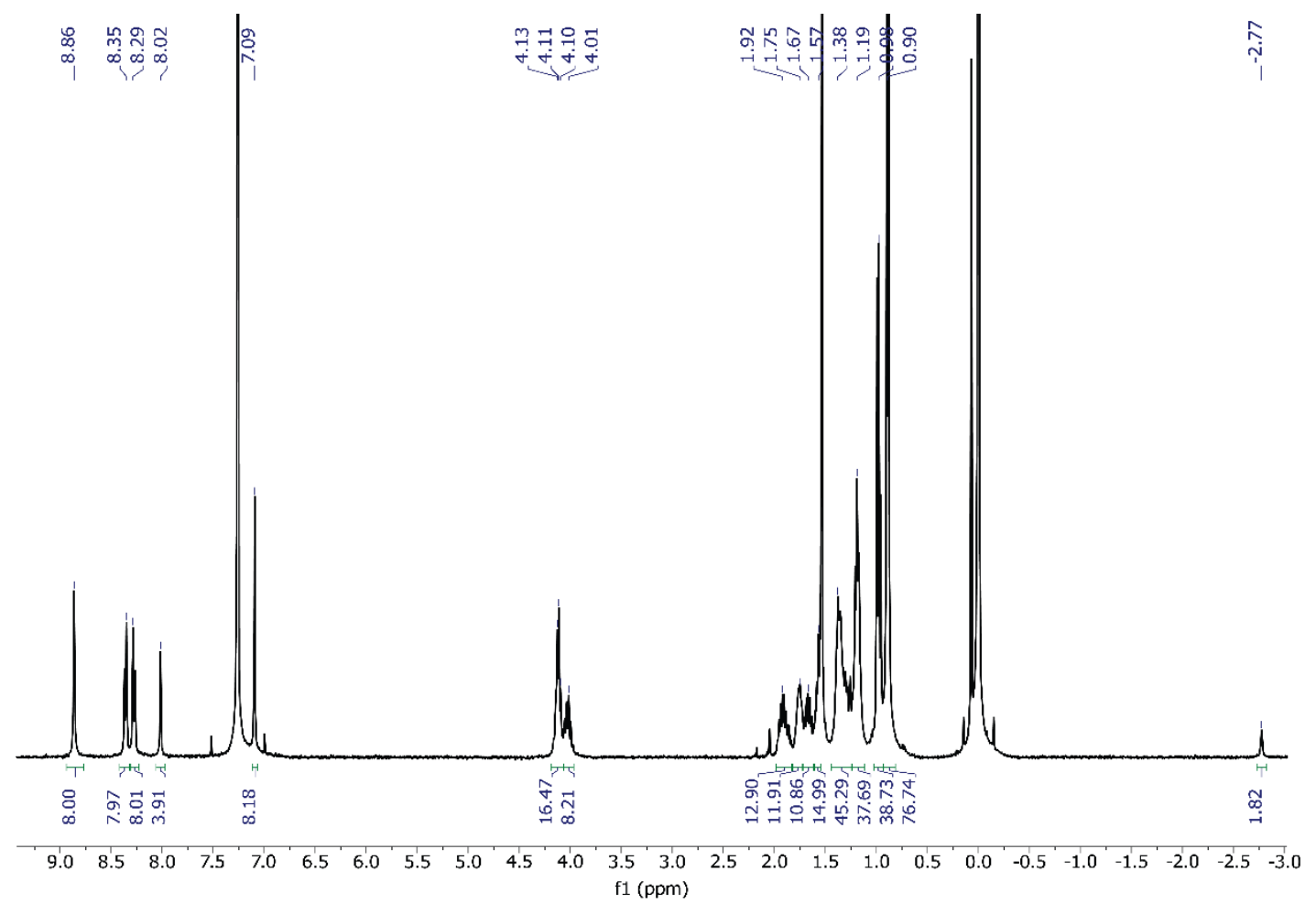

Figure $\mathrm{S} 1{ }^{1} \mathrm{H}$ NMR spectrum of $\mathbf{H}_{\mathbf{2}} \mathbf{- 1}$.
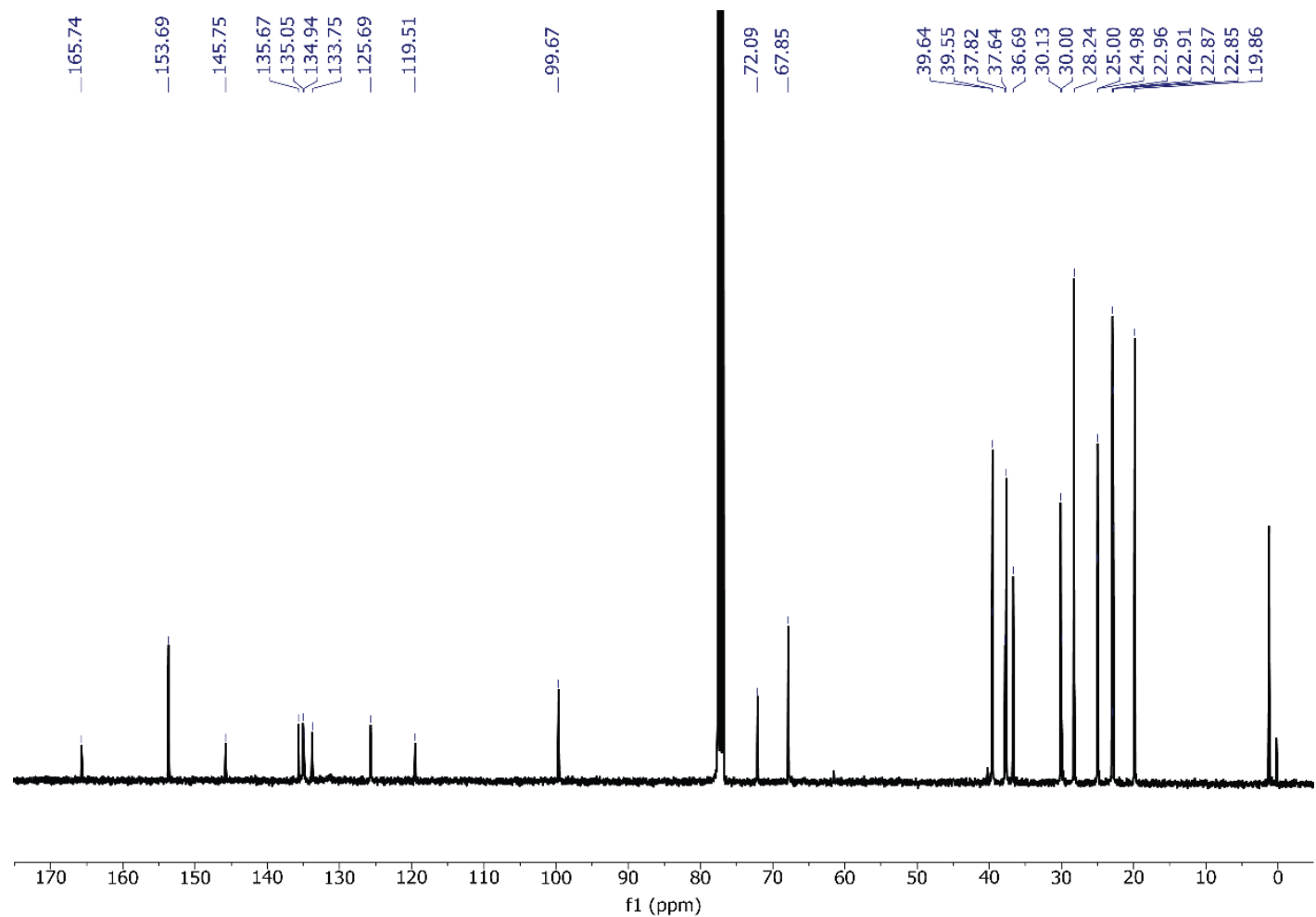

Figure $\mathrm{S} 2{ }^{13} \mathrm{C}$ NMR spectrum of $\mathbf{H}_{\mathbf{2}} \mathbf{- 1}$. 
${ }^{1} \mathrm{H}$ and ${ }^{13} \mathrm{C}$ NMR of MA

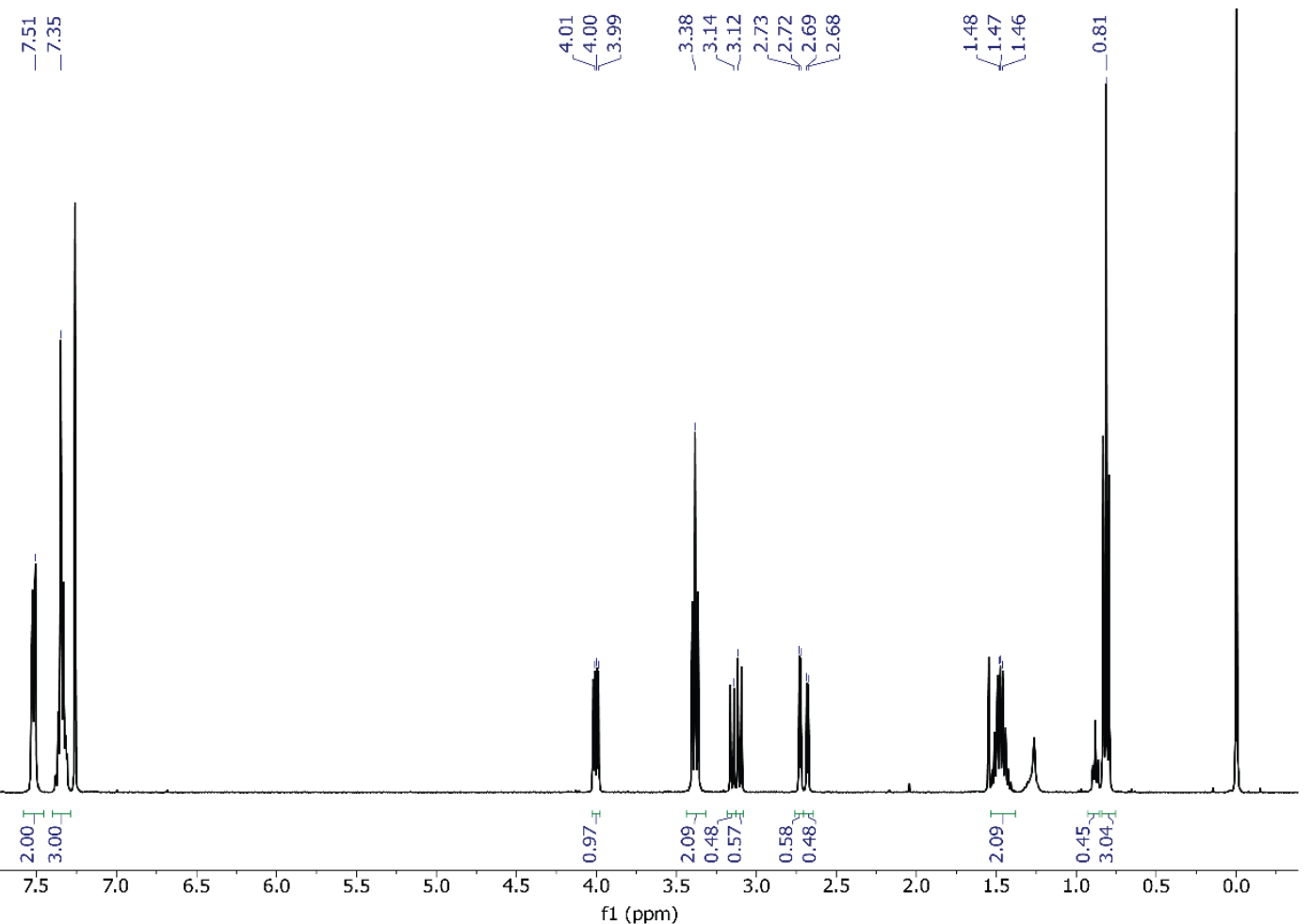

Figure $S 3{ }^{1} \mathrm{H}$ NMR spectrum of MA.
至

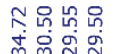

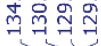

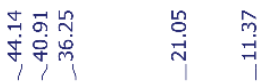

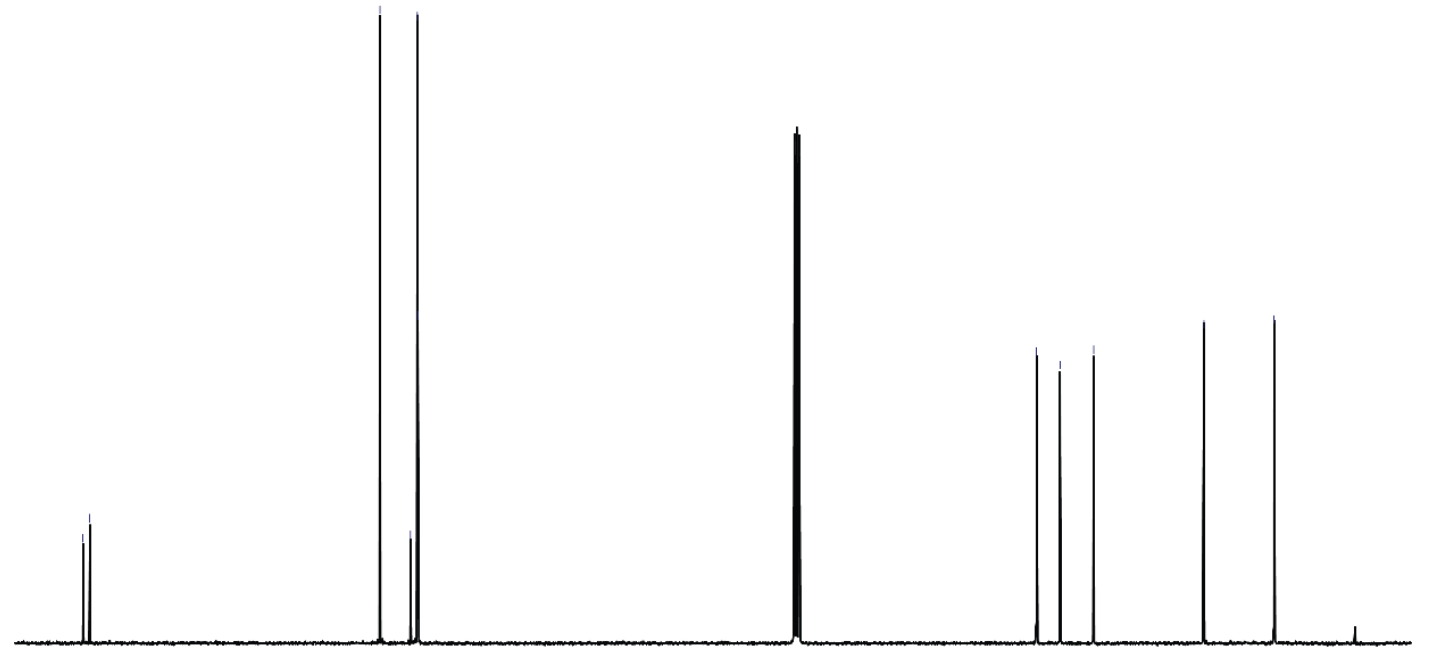

\begin{tabular}{lllllllllllllllllll}
\hline 180 & 170 & 160 & 150 & 140 & 130 & 120 & 110 & 100 & $\begin{array}{c}1 \\
\mathrm{f} 1(\mathrm{ppm})\end{array}$ & 80 & 70 & 60 & 50 & 40 & 30 & 20 & 10 & 0
\end{tabular}

Figure $S 4{ }^{13} \mathrm{C}$ NMR spectrum of MA. 
Spectra and titration curves of $\mathrm{Zn}-\mathrm{Me}$ with various piperidine catalysts<smiles>C1CCNC1</smiles>

$K_{\mathrm{a}}=949 \cdot 10^{3} \mathrm{M}^{-1}$

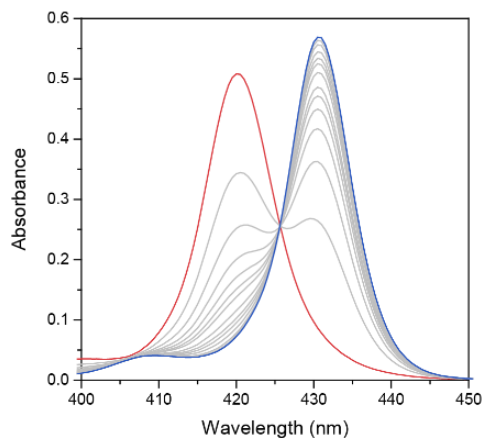<smiles>CC1CCCCN1</smiles>

$K_{a}=20.6 \cdot 10^{3} \mathrm{M}^{-1}$
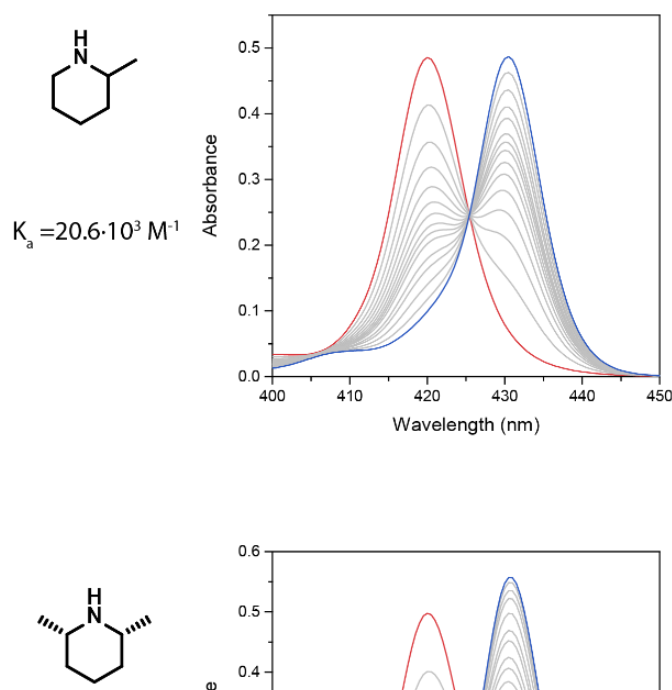

$\mathrm{K}_{\mathrm{a}}=6.63 \cdot 10^{3} \mathrm{M}^{-1}$
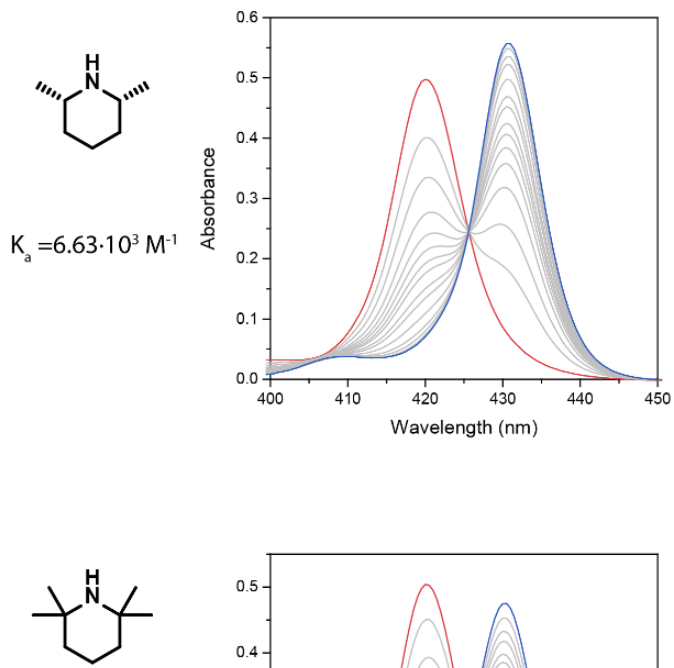

$K_{a}=168 M^{-1}$

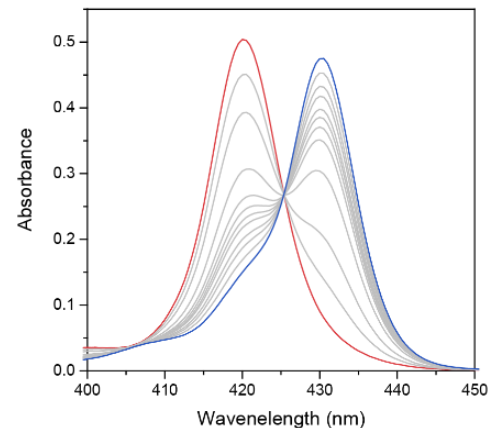

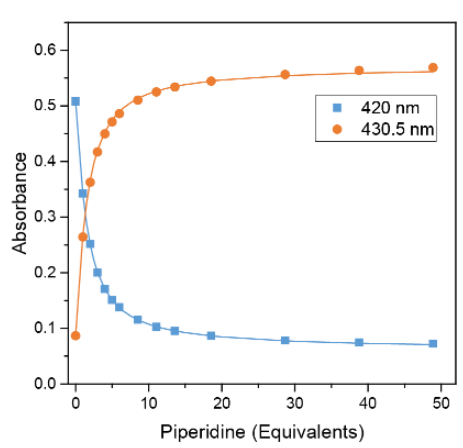
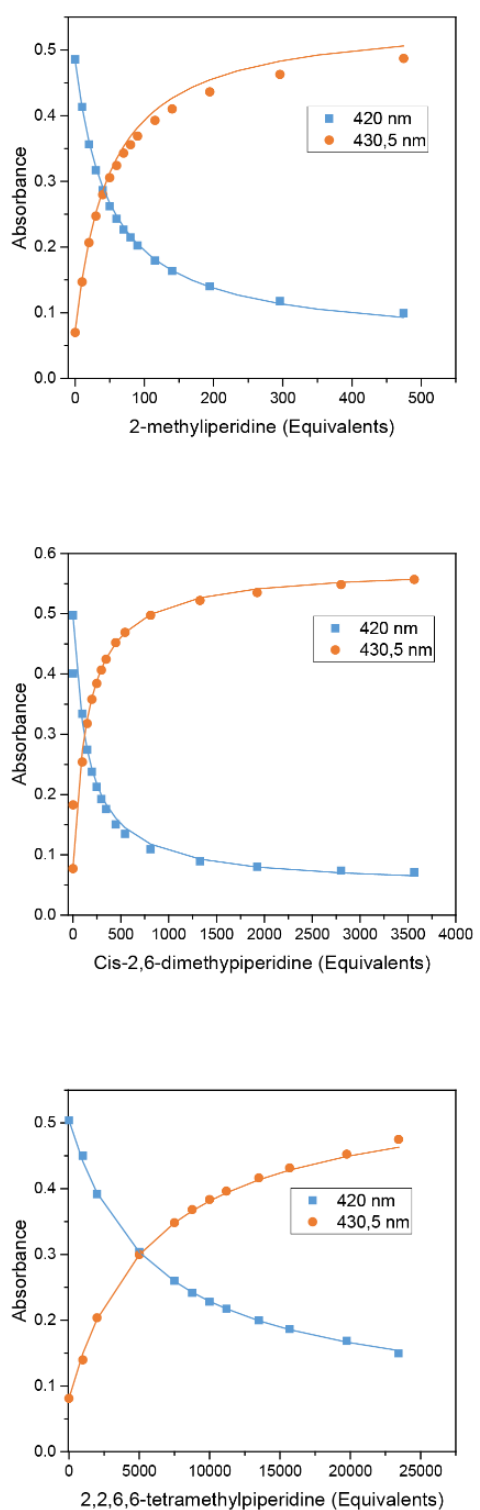

Figure S5 Spectra and titration curves of $1 \mu \mathrm{M}$ Zn-Me measured in $\mathrm{MCH}$ with the different piperidine based catalysts. The blue spectra indicate the spectra without ligand and the red spectra indicate the spectra with maximum amine concentration. 


\section{Time dependent $\mathrm{CD}$ spectra of $\mathrm{Zn}-1$ in the reaction mixture in dry, ambient and wet $\mathrm{MCH}$}

a)

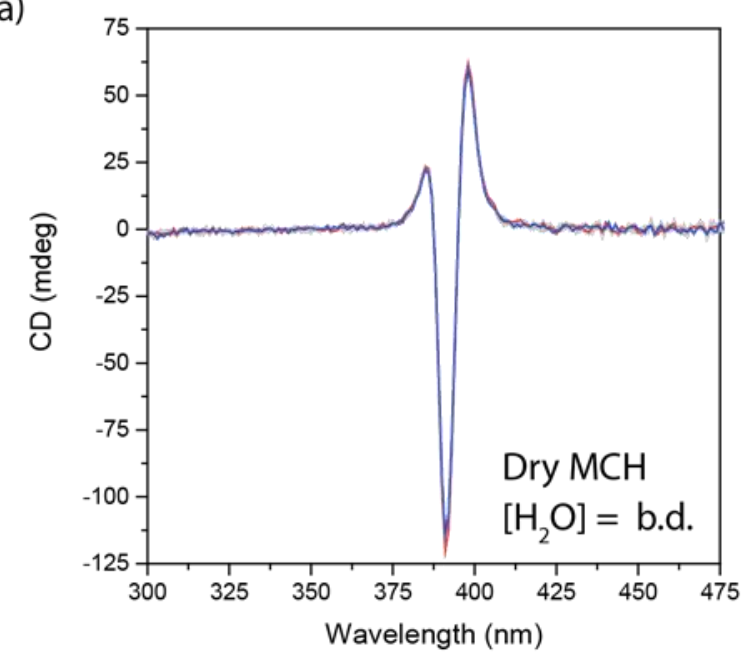

c)

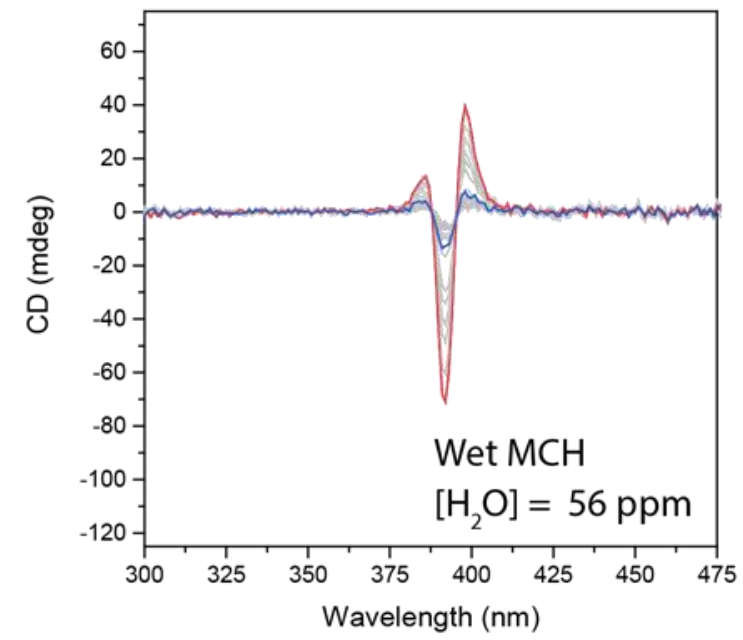

b)

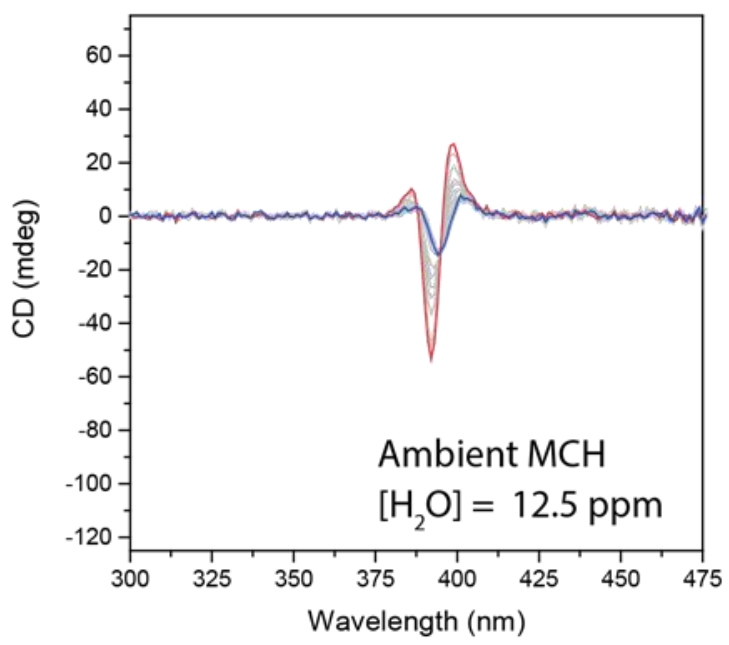

Figure S6 Selection of CD spectra for samples with $10 \mu \mathrm{M}$ Zn-1 and 0.5 mM Me2Pip, 4 mM NPrMal and 4 mM PhSH in dry (a), ambient (b) and wet (c) $\mathrm{MCH}$, collected together with the UV-Vis spectra shown in Figure 2 in the main text and Figure S7 in the Supporting Information. In the dry sample, the concentration of water is below the detection limit of the Karl-Fisher titrator. The red spectra indicate the first collected spectrum and the blue spectrum indicate the last collected spectra, corresponding to the last time point in Figures $2 b, 2 d$ and S7b. 


\section{Time-dependent UV-Vis results of $\mathrm{Zn}-1$ in the Michael reaction mixture in wet $\mathrm{MCH}$}

a)

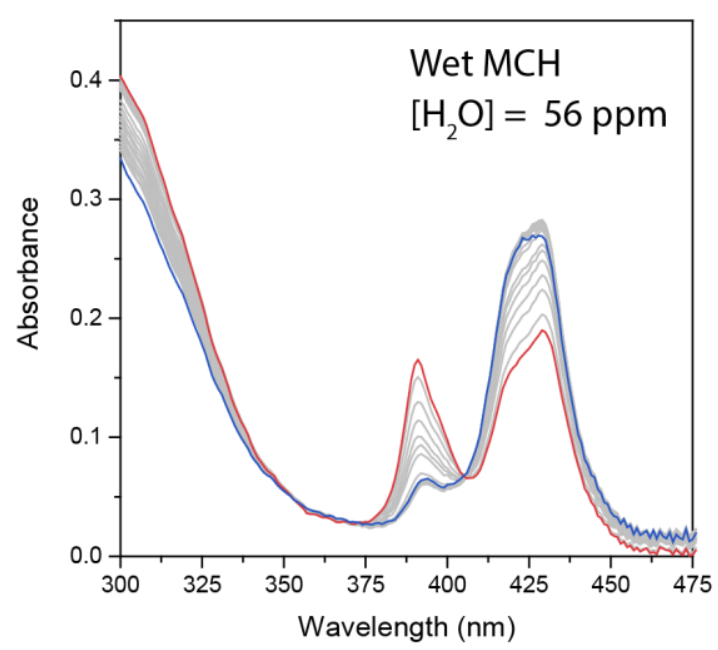

b)

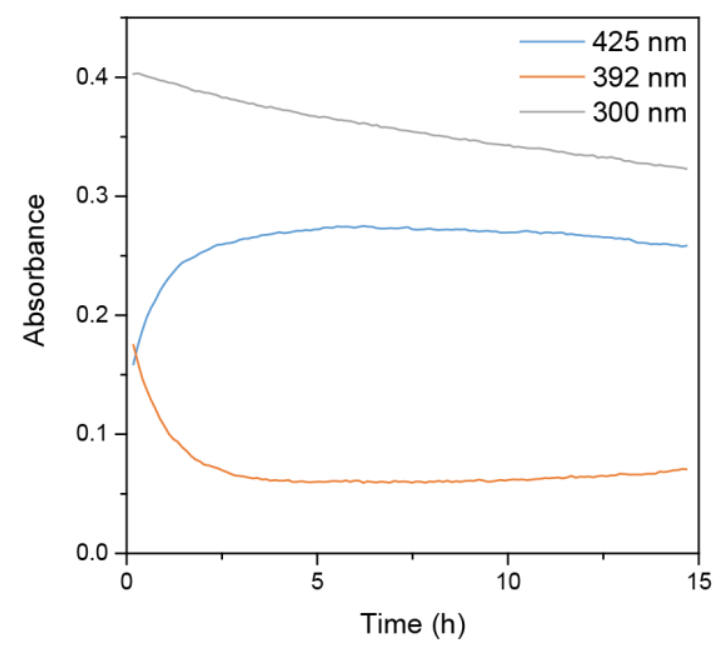

Figure S7 a) Selection of UV-Vis spectra of a solution of $10 \mu \mathrm{M}$ Zn-1 with $0.5 \mathrm{mM} \mathrm{MePip}$ and $4 \mathrm{mM}$ NPrMal and PhSH in wet MCH between 0 (red spectrum) and 15 hours (blue spectrum) of the measurement. b) Time dependency of the absorbance at $300 \mathrm{~nm}$, $392 \mathrm{~nm}$, and $425 \mathrm{~nm}$. 


\section{Time-dependent UV-Vis result of Zn-1 with PhSH and NPrMal in dry $\mathrm{MCH}$}

a)
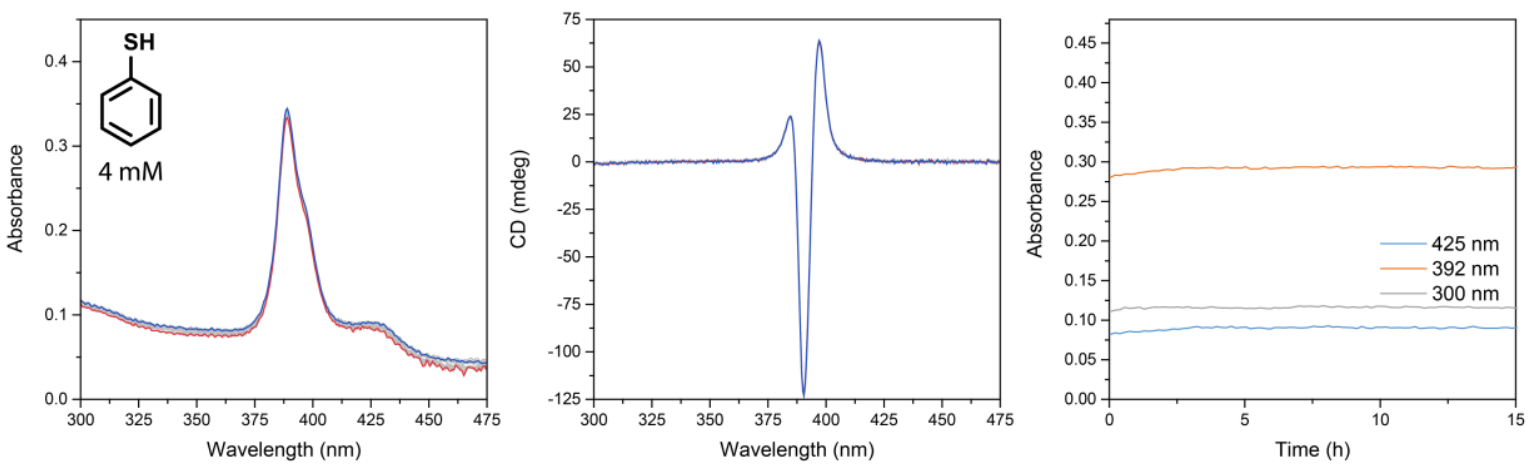

b)
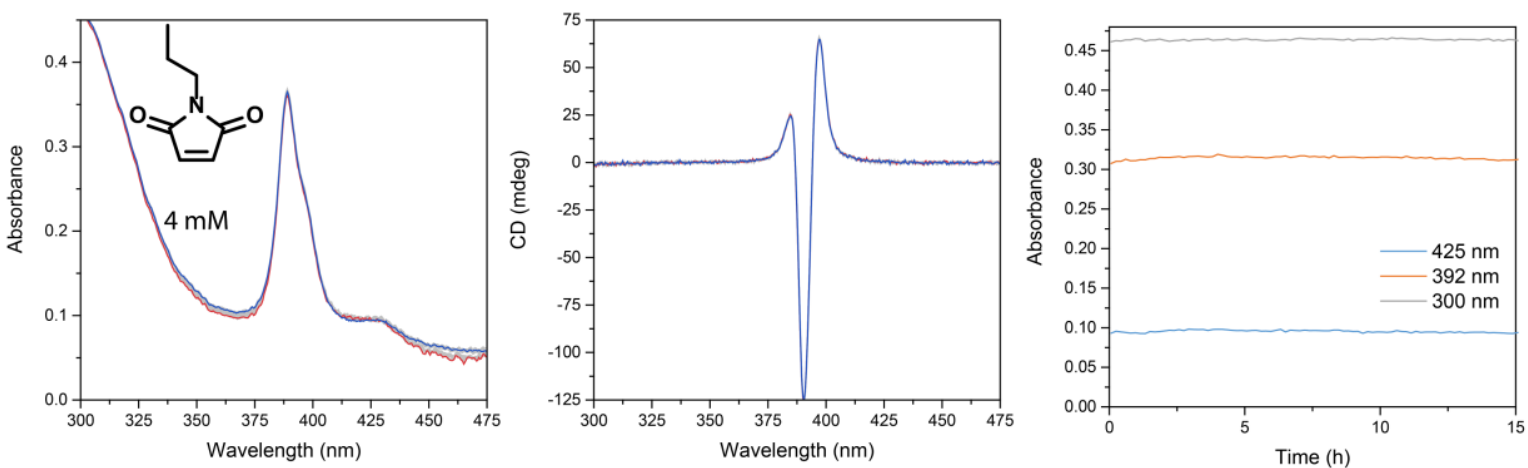

Figure S8 Selection of UV-Vis spectra (left panels) and simultaneously collected CD spectra (middle panels) of samples of $10 \mu \mathrm{M}$ Zn-1 with $4 \mathrm{mM} \mathrm{PhSH} \mathrm{(a)} \mathrm{and} 4 \mathrm{mM}$ NPrMal (b) in dry $\mathrm{MCH}\left(\left[\mathrm{H}_{2} \mathrm{O}\right]=9.0 \mathrm{ppm}\right)$. The spectra were collected between 0 hours (red spectrum) and 15 hours (blue spectrum) after sample preparation. The right panels show the time dependency of the absorbance at $300 \mathrm{~nm}, 392 \mathrm{~nm}$ and $425 \mathrm{~nm}$, which is extracted from the spectra collected at various time points. 
a)

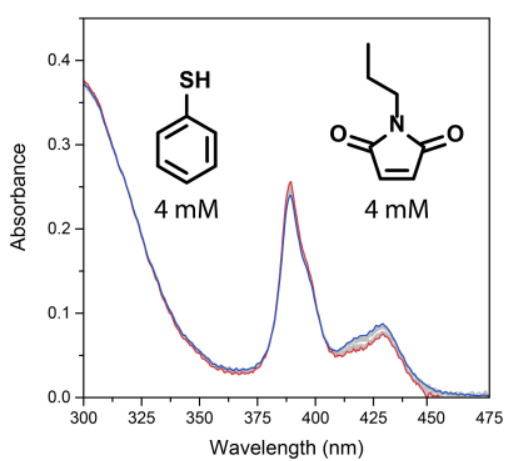

b)

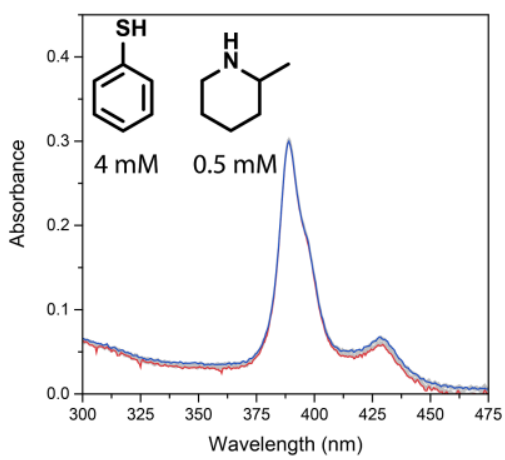

c)

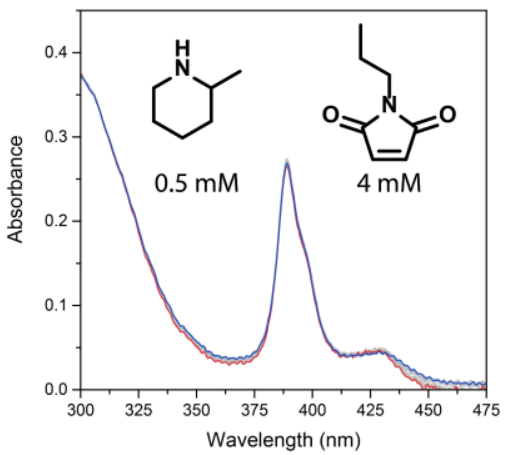

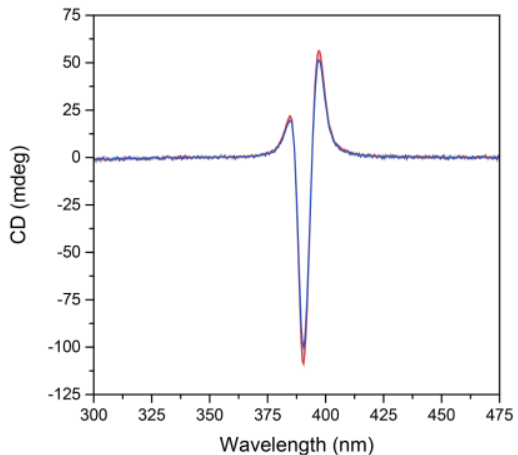
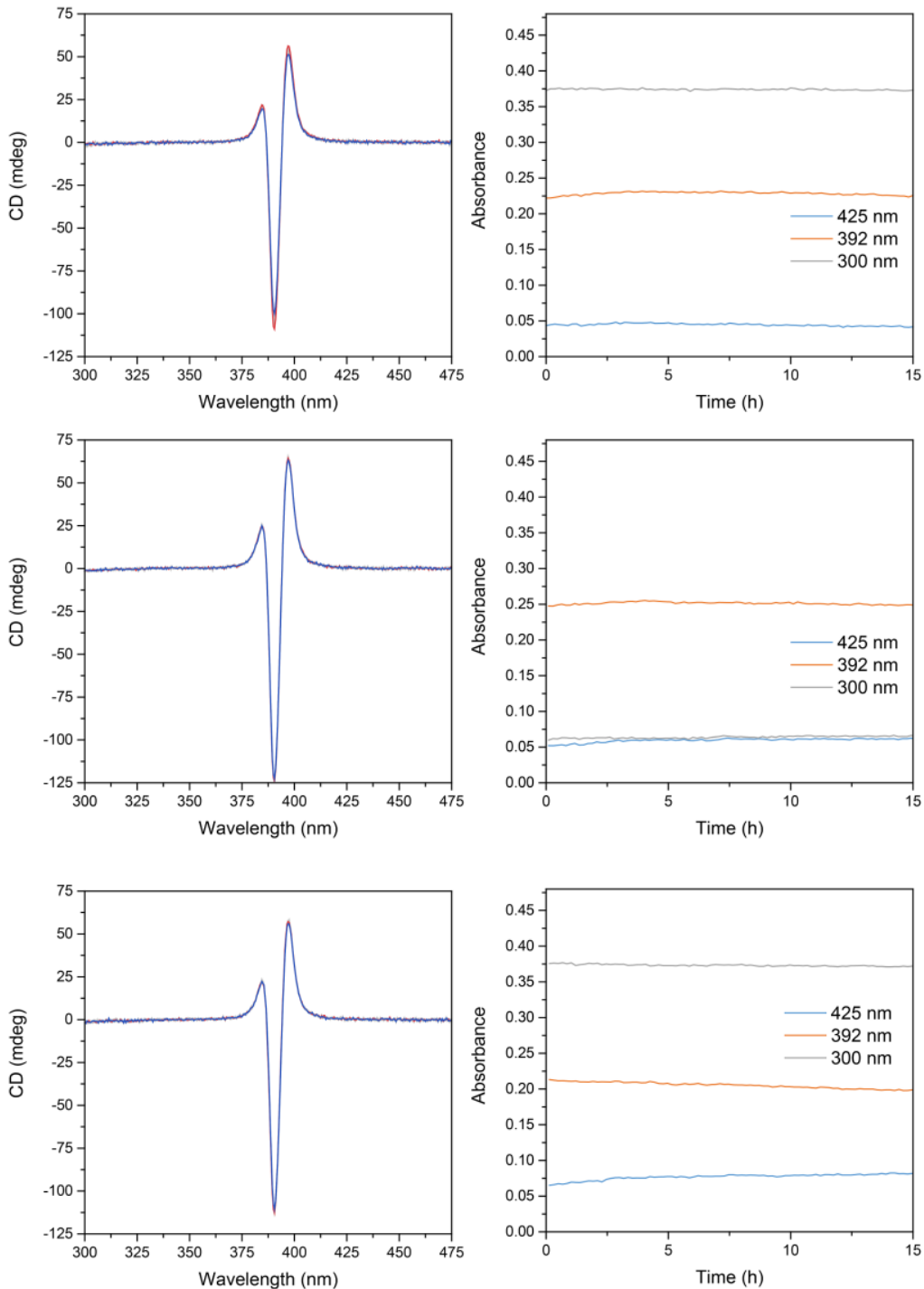

Figure S9 Selection of UV-Vis spectra (left panels) and simultaneously collected CD spectra (middle panels) of samples of $10 \mu \mathrm{M}$ Zn-1 with $4 \mathrm{mM}$ PhSH and $4 \mathrm{mM}$ NPrMal (a), $4 \mathrm{mM}$ PhSH with $0.5 \mathrm{mM}$ MePip (b) and $4 \mathrm{mM}$ NPrMal with $0.5 \mathrm{mM}$ MePip in ambient $\mathrm{MCH}\left(\left[\mathrm{H}_{2} \mathrm{O}\right]=9.0 \mathrm{ppm}\right)$. The spectra were collected between 0 hours (red spectrum) and 15 hours (blue spectrum) after sample preparation. The right panels show the time dependency of the absorbance at $300 \mathrm{~nm}, 392 \mathrm{~nm}$ and $425 \mathrm{~nm}$, which is extracted from the spectra collected at various time points. 
Time-dependent UV-Vis \& CD result of Zn-1 with PhSH and NPrMal in ambient $\mathrm{MCH}$

a)
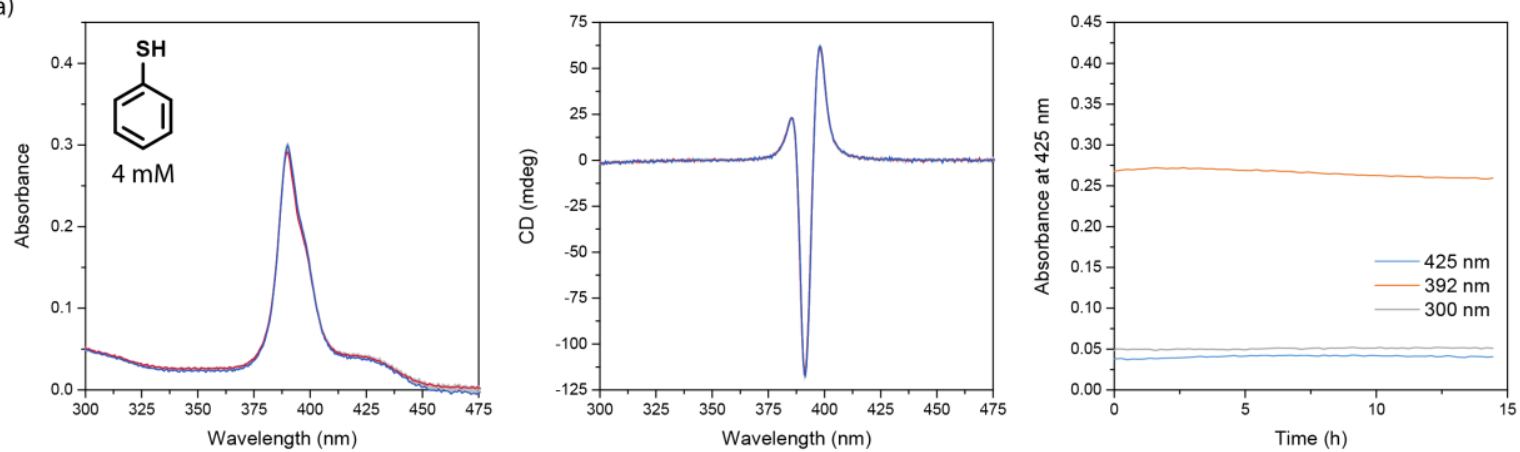

b)
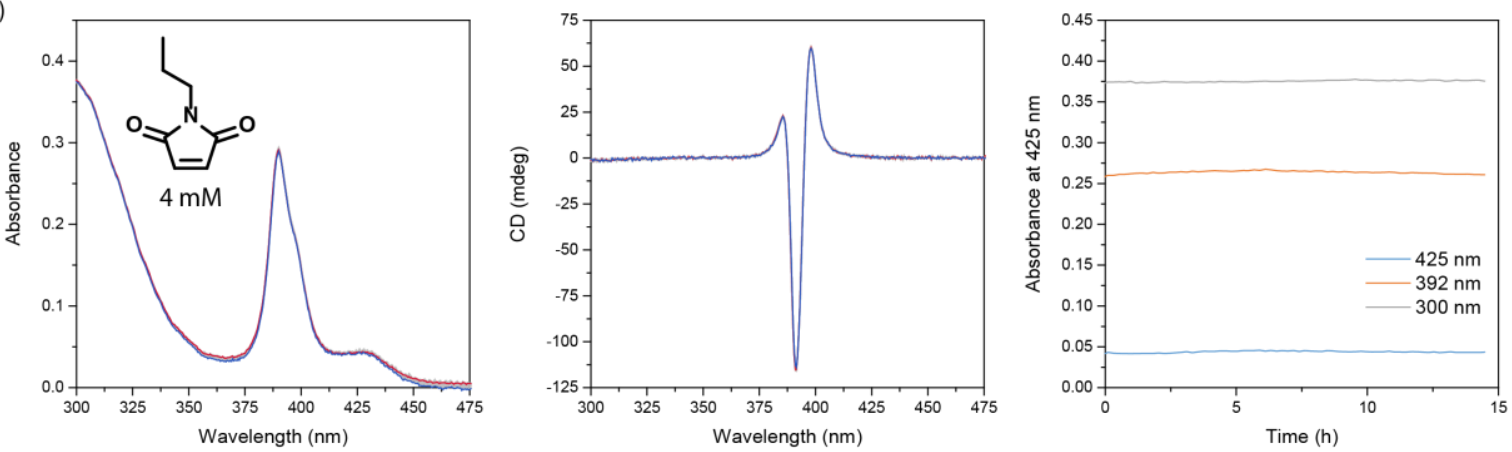

Figure S10 Selection of UV-Vis spectra (left panels) and simultaneously collected CD spectra (middle panels) of samples of $10 \mu \mathrm{M}$ $\mathrm{Zn}-1$ with $4 \mathrm{mM} \mathrm{PhSH} \mathrm{(a)} \mathrm{and} 4 \mathrm{mM}$ NPrMal (b) in ambient $\mathrm{MCH}\left(\left[\mathrm{H}_{2} \mathrm{O}\right]=12.5 \mathrm{ppm}\right)$. The spectra were collected between 0 hours (red spectrum) and 15 hours (blue spectrum) after sample preparation. The right panels show the time dependency of the absorbance at $300 \mathrm{~nm}, 392 \mathrm{~nm}$ and $425 \mathrm{~nm}$, which is extracted from the spectra collected at various time points. 
UV-Vis and CD spectra titrations Zn-1 with combinations of PhSH, NPrMal and MePip in ambient $\mathrm{MCH}$

a)
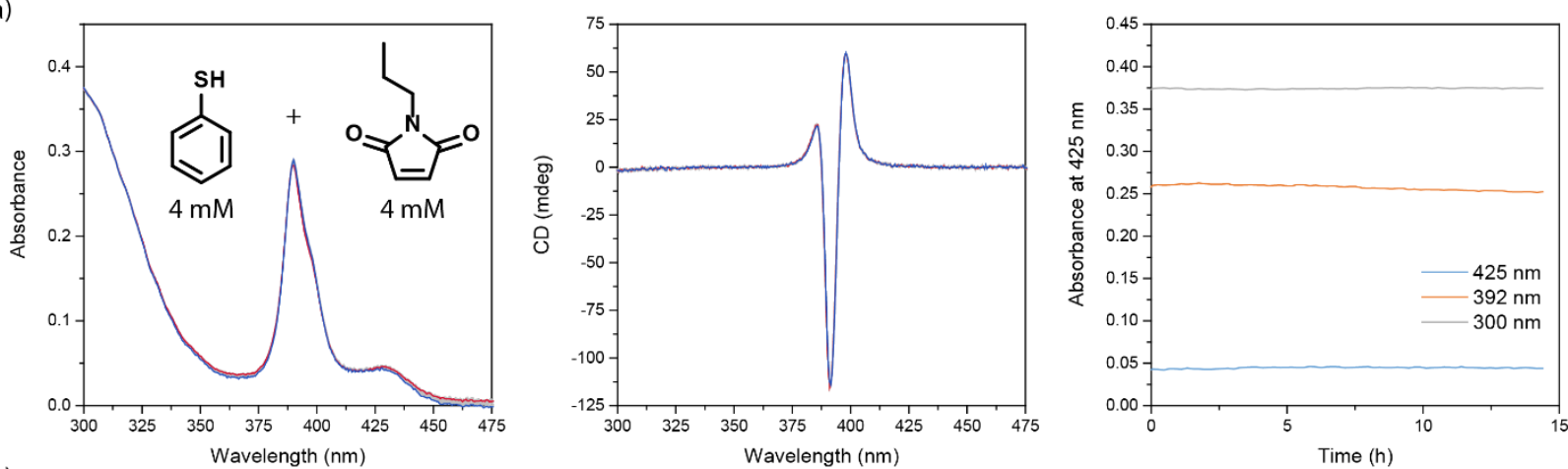

b)
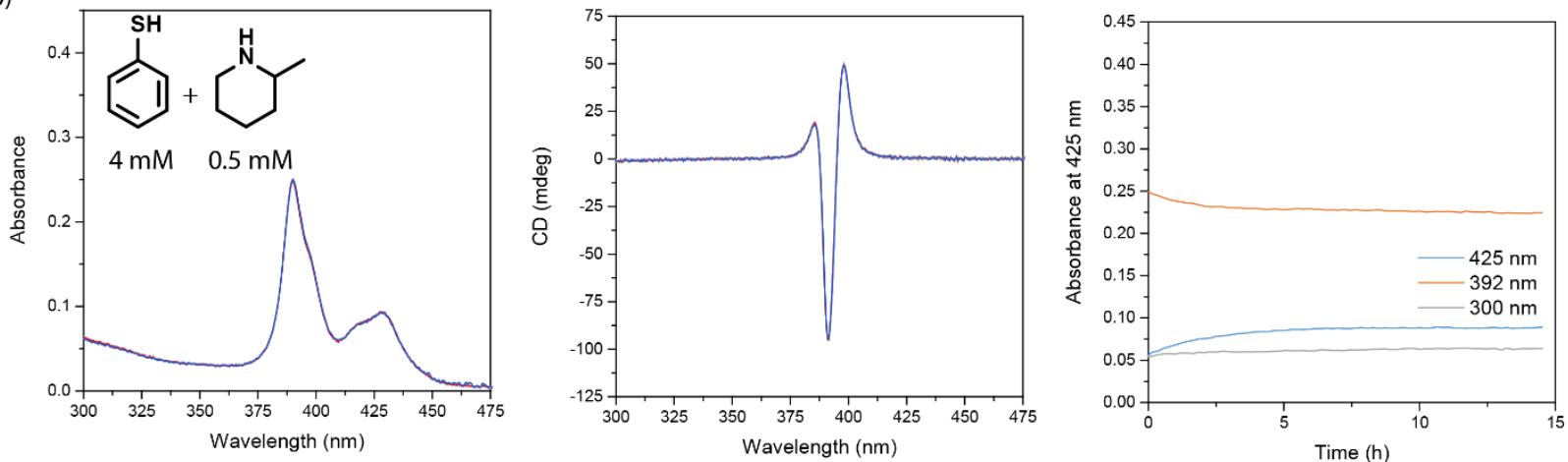

c)
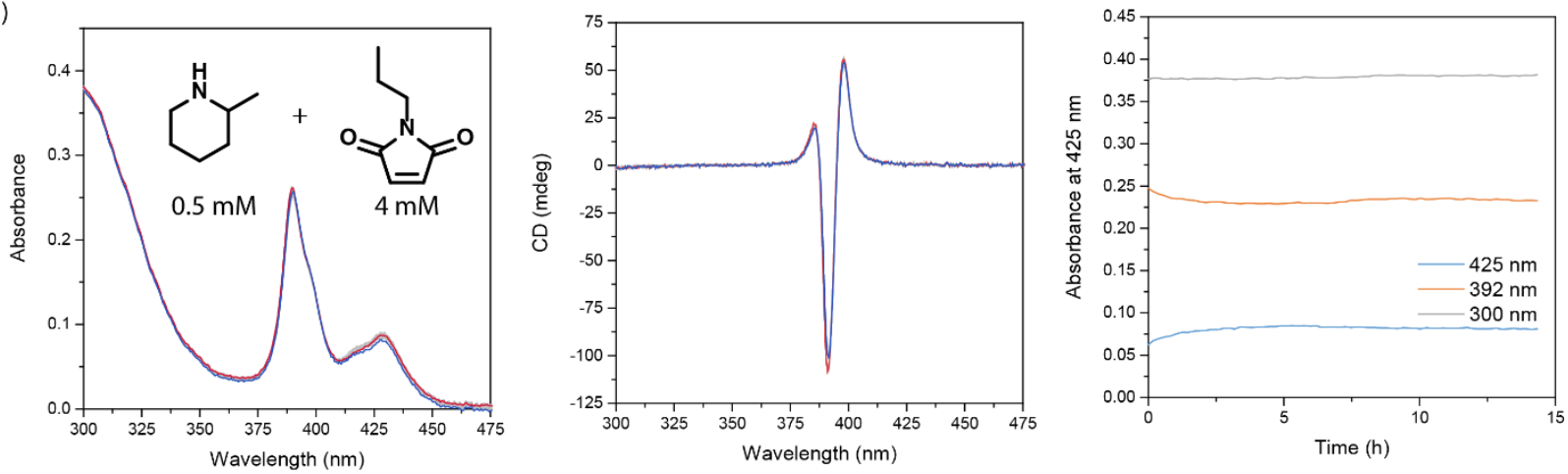

Figure S11 Selection of UV-Vis spectra (left panels) and simultaneously collected CD spectra (middle panels) of samples of $10 \mu \mathrm{M}$ Zn-1 with $4 \mathrm{mM}$ PhSH and $4 \mathrm{mM}$ NPrMal (a), $4 \mathrm{mM}$ PhSH with $0.5 \mathrm{mM}$ MePip (b) and $4 \mathrm{mM}$ NPrMal with $0.5 \mathrm{mM} \mathrm{MePip} \mathrm{in} \mathrm{ambient} \mathrm{MCH}$ $\left(\left[\mathrm{H}_{2} \mathrm{O}\right]=12.5 \mathrm{ppm}\right)$. The spectra were collected between 0 hours (red spectrum) and 15 hours (blue spectrum) after sample preparation. The right panels show the time dependency of the absorbance at $300 \mathrm{~nm}, 392 \mathrm{~nm}$ and $425 \mathrm{~nm}$, which is extracted from the spectra collected at various time points. 
AFM micrographs of $\mathrm{Zn}-1$ in dry and $\mathrm{MCH}^{*}$

\section{Dry}

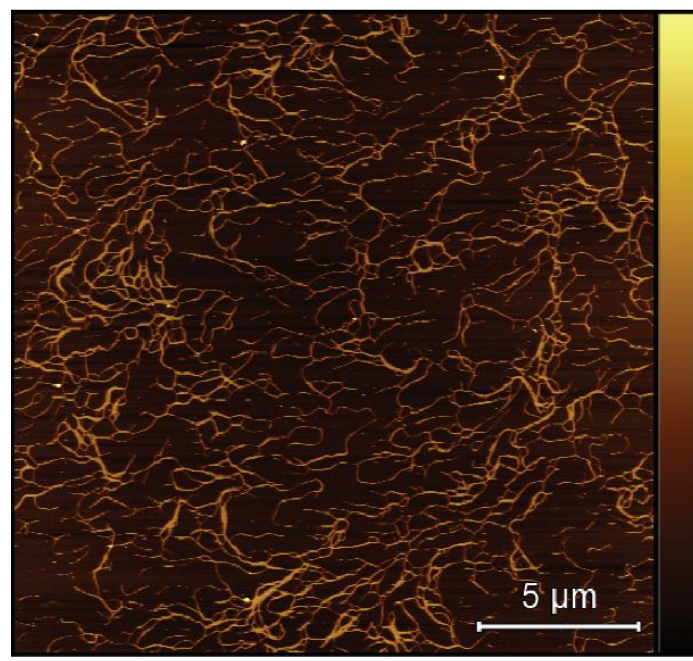

$6.0 \mathrm{~nm}$
5.0
4.0
3.0
2.0
1.0
0.0
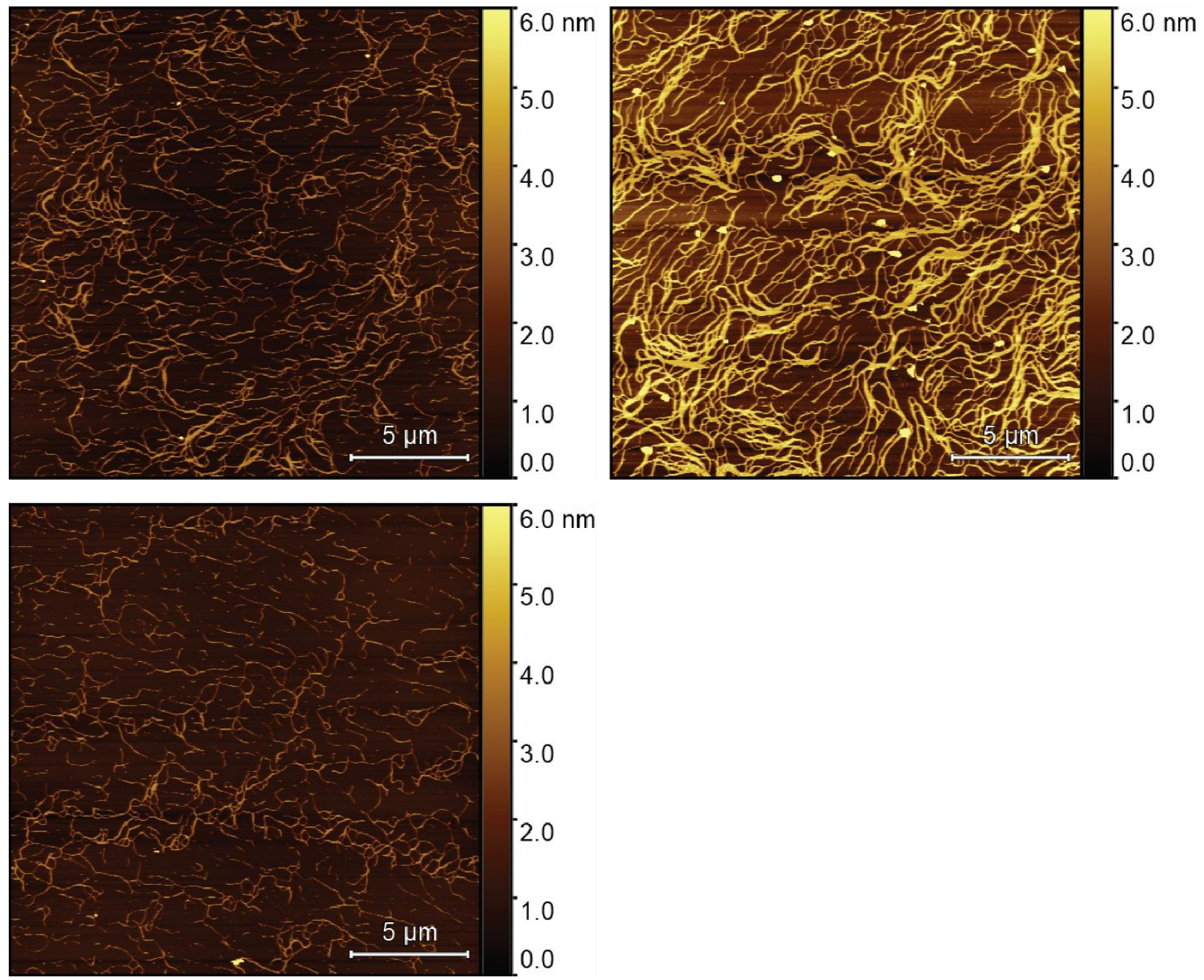

Figure S12 AFM micrographs of samples drop casted from solutions of $10 \mu \mathrm{M} \mathrm{Zn-1}$ in dry $\mathrm{MCH}^{*}$. [H $\left.\mathrm{H}_{2} \mathrm{O}\right]=20.0 \mathrm{ppm}$. 


\section{Ambient}
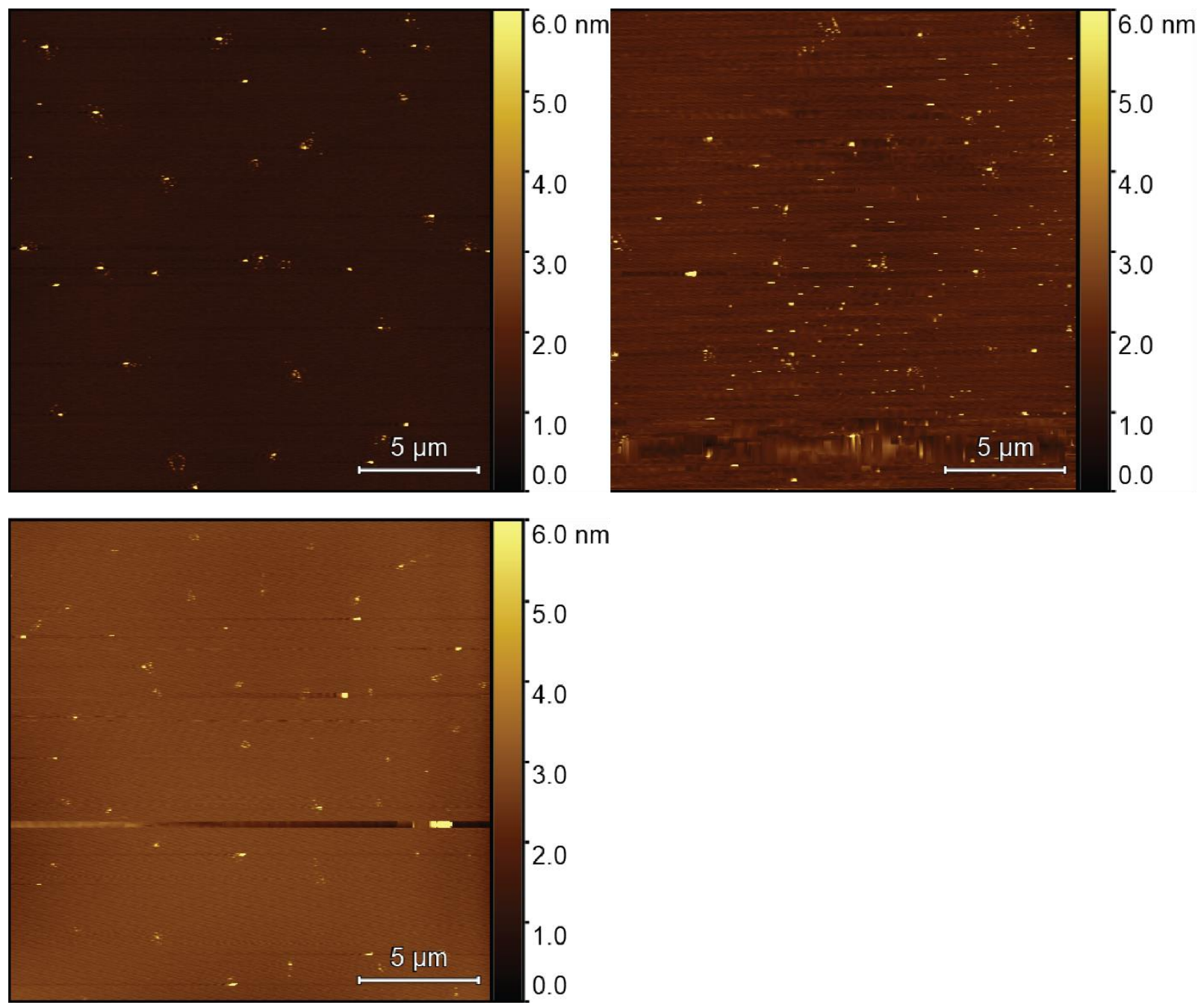

Figure S13 AFM micrographs of samples drop casted from solutions of $10 \mu \mathrm{M} \mathrm{Zn-1}$ in ambient $\mathrm{MCH}^{*}$. [ $\left.\mathrm{H}_{2} \mathrm{O}\right]=48.9 \mathrm{ppm}$. 


\section{SLS count rate at various concentrations of $\mathrm{Zn}-1$}

a)

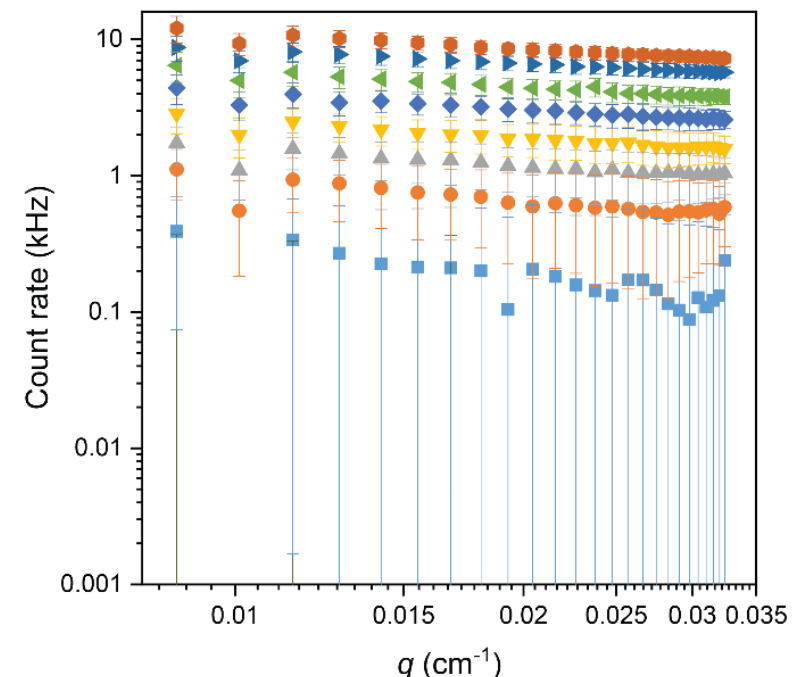

b)

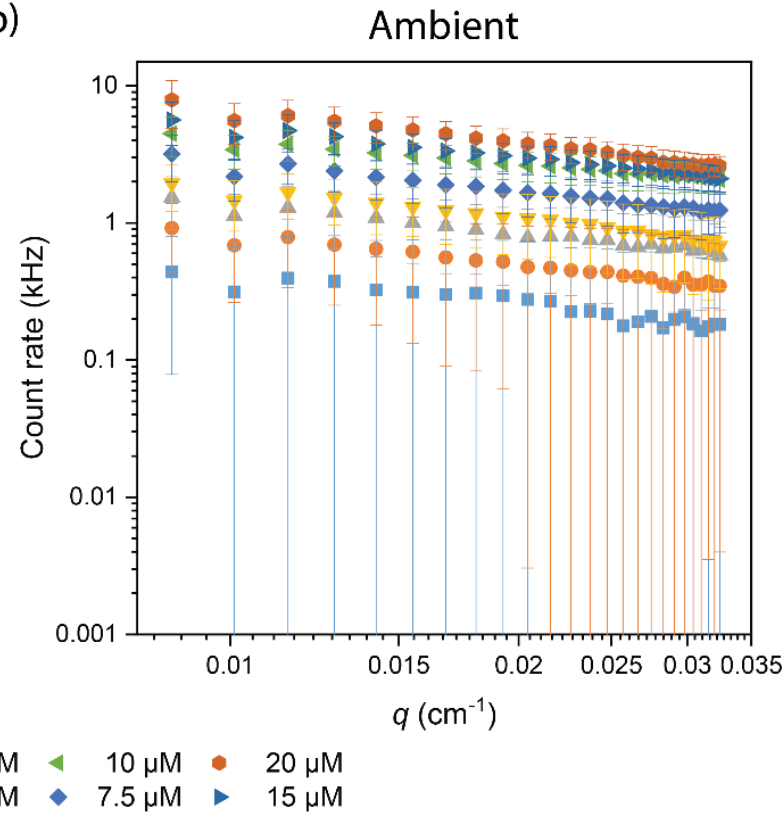

Figure S14 Light scattering count rates at various concentrations of $10 \mu \mathrm{M} \mathrm{Zn-1}$ in dry (a) and ambient (b) $\mathrm{MCH}^{*}$. In the dry samples, $\left[\mathrm{H}_{2} \mathrm{O}\right]=5.1 \mathrm{ppm}$ and in the ambient samples, $\left[\mathrm{H}_{2} \mathrm{O}\right]=52.1 \mathrm{ppm}$.

\section{Comparison SLS count rates at various concentrations and humidities}

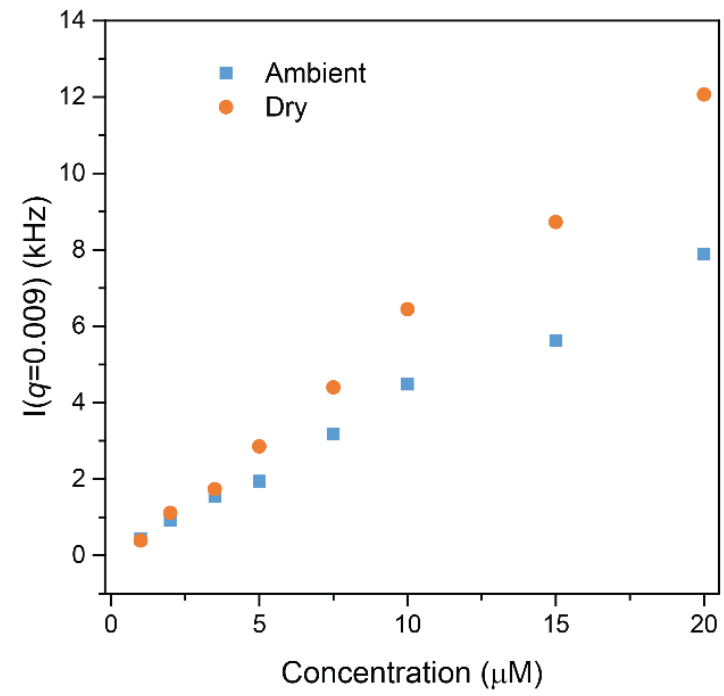

Figure S15 Light scattering count rates at $q=0.009 \mathrm{~cm}^{-1}$ at various concentrations of $10 \mu \mathrm{M} \mathrm{Zn-1}$ in dry (circles) and ambient (squares) $\mathrm{MCH}^{*}$. In the dry samples, $\left[\mathrm{H}_{2} \mathrm{O}\right]=5.1 \mathrm{ppm}$ and in the ambient samples, $\left[\mathrm{H}_{2} \mathrm{O}\right]=52.1 \mathrm{ppm}$. The linear increase indicates the polymer chains are in the dilute rather than semi-dilute regime. 


\section{UV-Vis spectra of $\mathrm{Zn-1}$ after filtering through various filters}

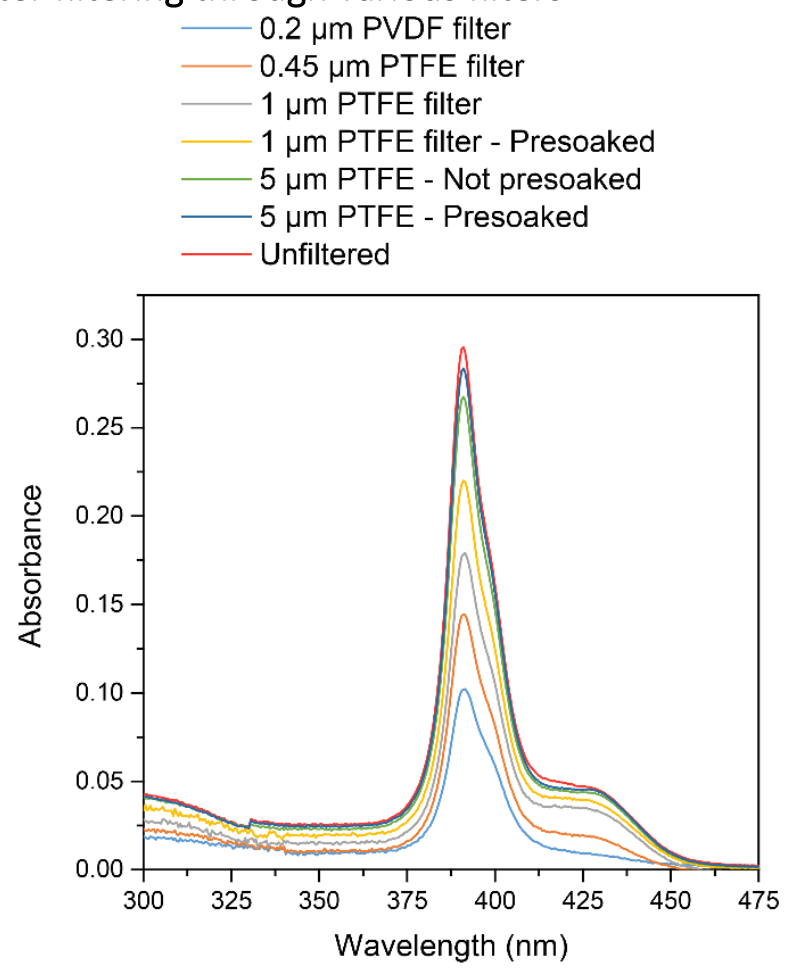

Figure S16 UV-Vis spectra of $10 \mu \mathrm{M}$ solutions of $\mathbf{Z n - 1}$ in ambient $\mathrm{MCH}^{*}$ after filtering through various filters. The $0.2 \mu \mathrm{m}$ and $0.45 \mu \mathrm{m}$ filter were not washed and presoaked with the Zn-1 solution before collection of the filtrate. For the $1 \mu \mathrm{m}$ and $5 \mu \mathrm{m}$ filters, both cases are shown. As can be seen, presoaking the filters with $1.5 \mathrm{~mL} \mathbf{Z n - 1}$ solution decreases adsorption to the filters and the presoaked $5 \mu \mathrm{m}$ filters pass almost all Zn-1 material.

Titration results of the titration of $\mathrm{Zn}-1$ with MA in the absence and presence of MePip in ambient and dry $\mathrm{MCH}^{*}$

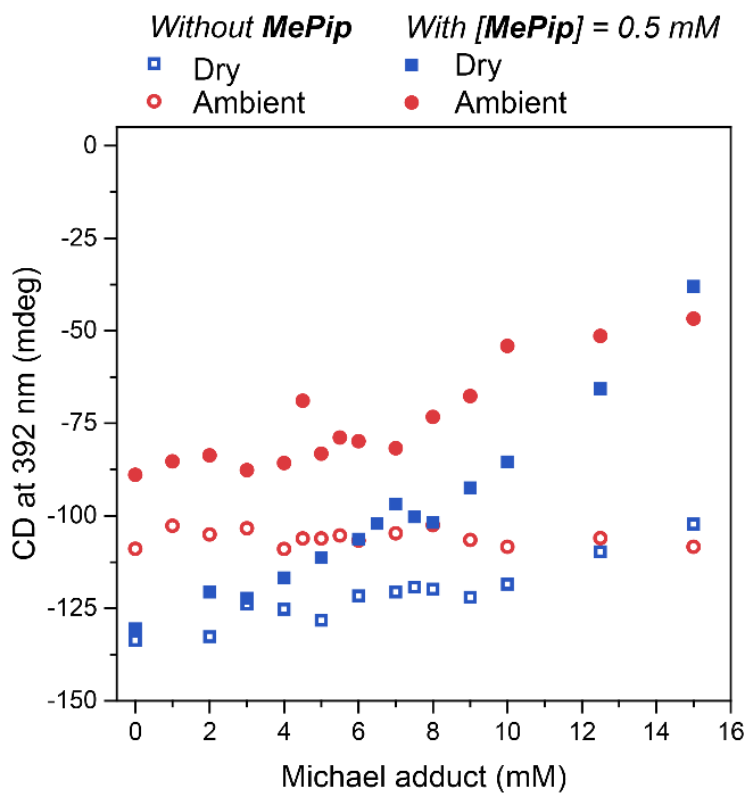

Figure S17 Titration results of the titration of $10 \mu \mathrm{M} \mathrm{Zn-1}$ with $\mathbf{M A}$ in dry ([ $\left.\mathrm{H}_{2} \mathrm{O}\right]$ below detection limit of the Karl-Fisher titatrator) and ambient $\left(\left[\mathrm{H}_{2} \mathrm{O}\right]=13.8 \mathrm{ppm}\right) \mathrm{MCH}^{*}$ in the presence and absence of $0.5 \mathrm{mM}$ MePip. 
Titration results of the titration of $\mathrm{Zn}-1$ with NPrMal in the presence of PhSH in ambient and dry $\mathrm{MCH}^{*}$

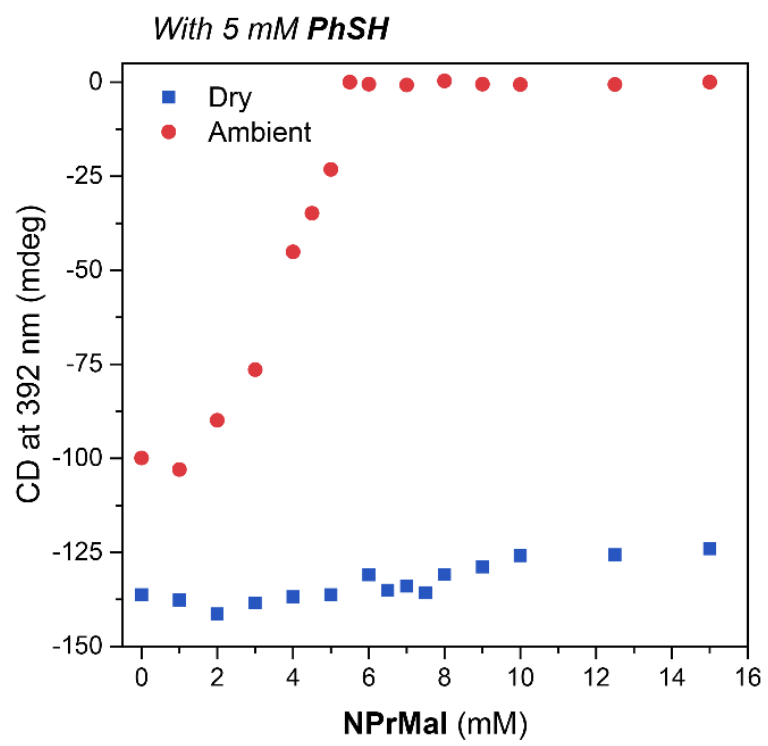

Figure S18 Titration results of the titration of $10 \mu \mathrm{M} \mathrm{Zn-1}$ with NPrMal in dry $\left(\left[\mathrm{H}_{2} \mathrm{O}\right]\right.$ below detection limit of the Karl-Fisher titatrator) and ambient $\left(\left[\mathrm{H}_{2} \mathrm{O}\right]=13.8 \mathrm{ppm}\right) \mathrm{MCH}^{*}$ in the presence of $5 \mathrm{mM} \mathrm{PhSH}$.

\section{UV-Vis and CD spectra of the titrations Zn-1 with PhSH, NPrMal and MA in ambient MCH*}

a)

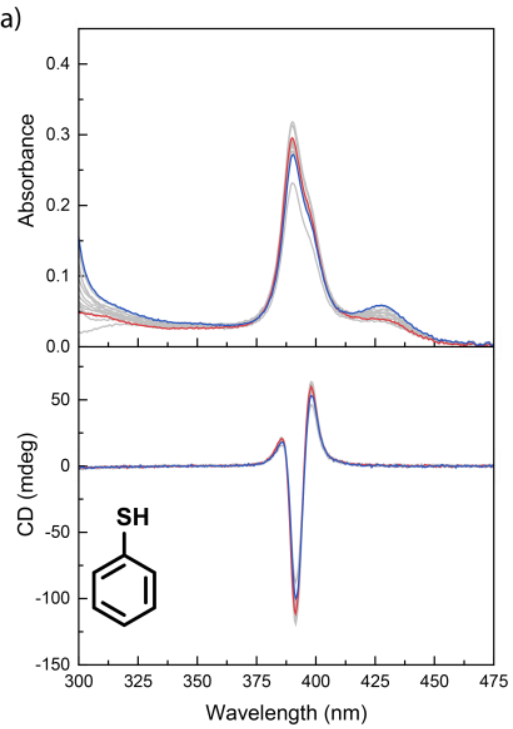

b)

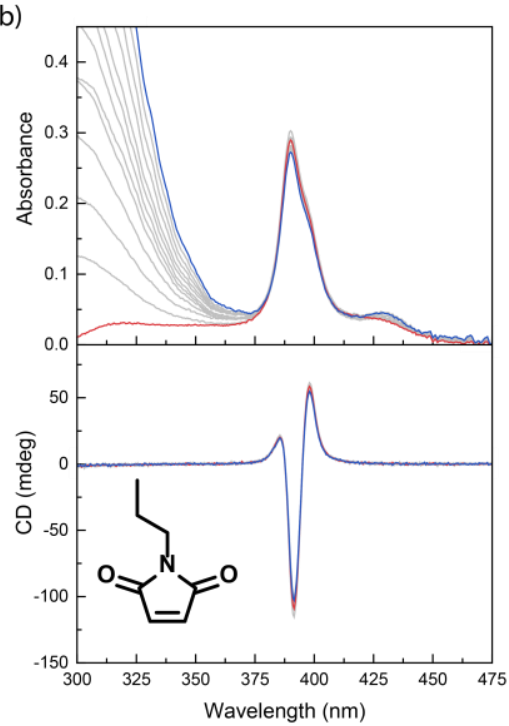

c)

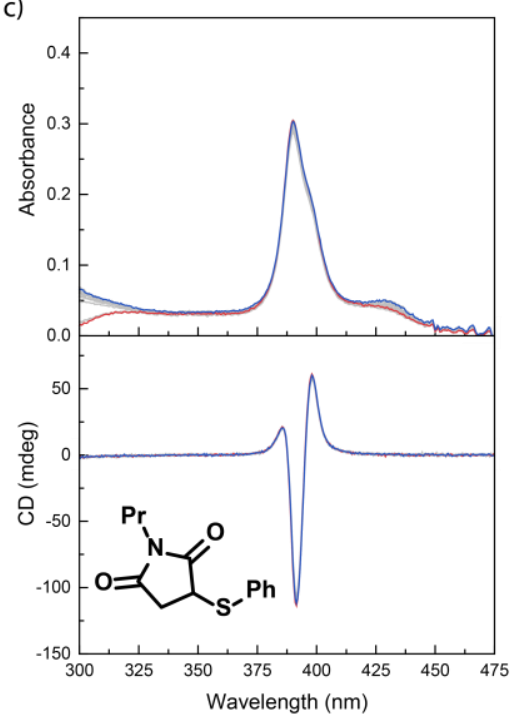

Figure S19 UV-Vis (top panels) and CD (bottom panels) spectra corresponding to the titrations of $10 \mu \mathrm{M}$ Zn-1 with PhSH, NPrMal and $\mathbf{M A}$ in ambient $\mathrm{MCH}^{*}$ and in the absence of MePip. From the spectra, the results given in Figure 4 in the main text and Figure S17 in the Supporting Information are obtained. The red spectra indicate the spectra without additive, while the blue spectra indicate the spectra collected at maximum additive concentration. 
UV-Vis and CD spectra of the titrations Zn-1 with the PhSH, NPrMal and MA in the presence of 0.5 $\mathrm{mM}$ MePip in ambient $\mathrm{MCH}^{*}$
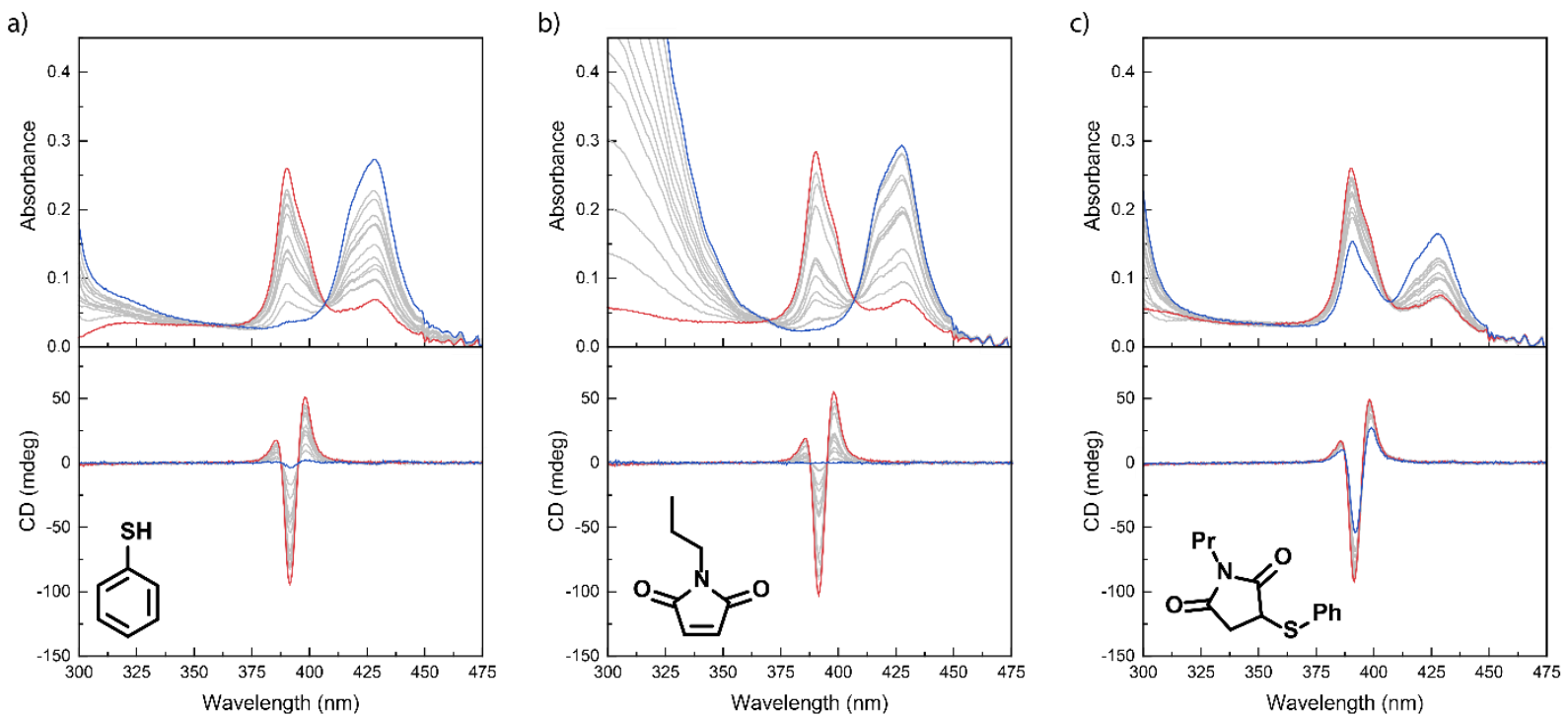

Figure S20 UV-Vis (top panels) and CD (bottom panels) spectra corresponding to the titrations of $10 \mu \mathrm{M}$ Zn-1 with PhSH, NPrMal and $\mathrm{MA}$ in ambient $\mathrm{MCH}^{*}$ and in the presence of $0.5 \mathrm{mM}$ MePip. From the spectra, the results given in Figure 4 in the main text and Figure S17 in the Supporting Information are obtained. The red spectra indicate the spectra without additive, while the blue spectra indicate the spectra collected at maximum additive concentration. 
UV-Vis and CD spectra of the titrations $\mathrm{Zn}-1$ with NPrMal in the presence of $5 \mathrm{mM} \mathrm{PhSH}$ in ambient $\mathrm{MCH}^{*}$

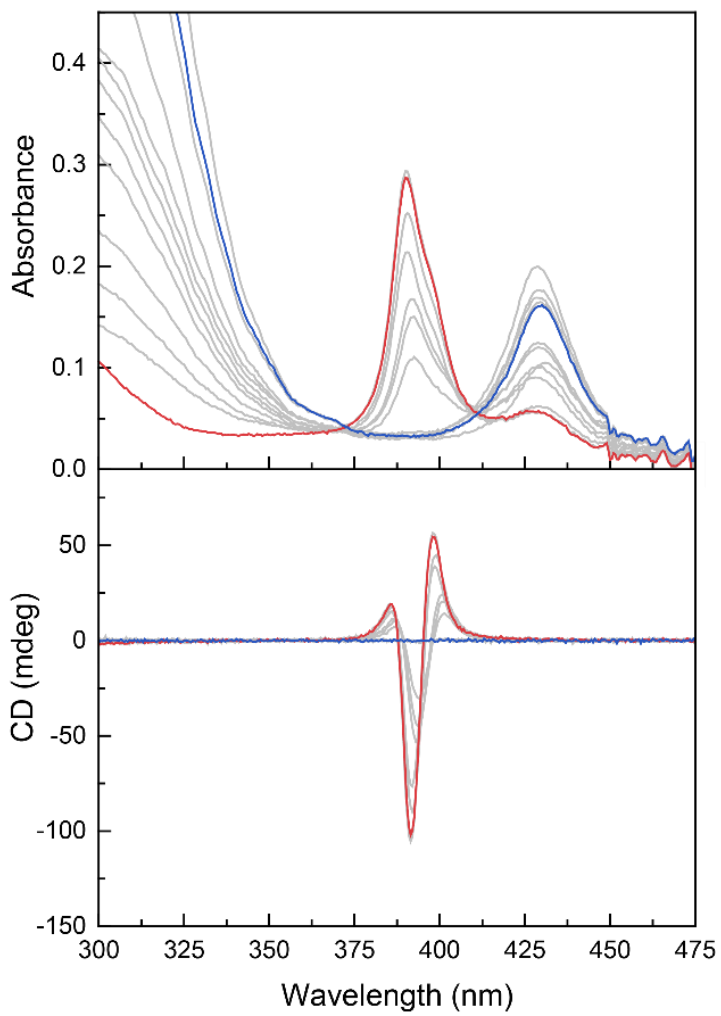

Figure S21 UV-Vis (top panel) and CD (bottom panels) spectra corresponding to the titrations of Zn-1 with NPrMal in ambient $\mathrm{MCH}^{*}$ and in the presence of $5 \mathrm{mM}$ PhSH. From the spectra, the results given in Figure S18 in the Supporting Information are obtained. The red spectra indicate the spectra without additive, while the blue spectra indicate the spectra collected at maximum additive concentration. 


\section{UV-Vis and CD spectra of the titrations $\mathrm{Zn}-1$ with $\mathrm{PhSH}, \mathrm{NPrMal}$ and MA in dry $\mathrm{MCH}^{*}$}
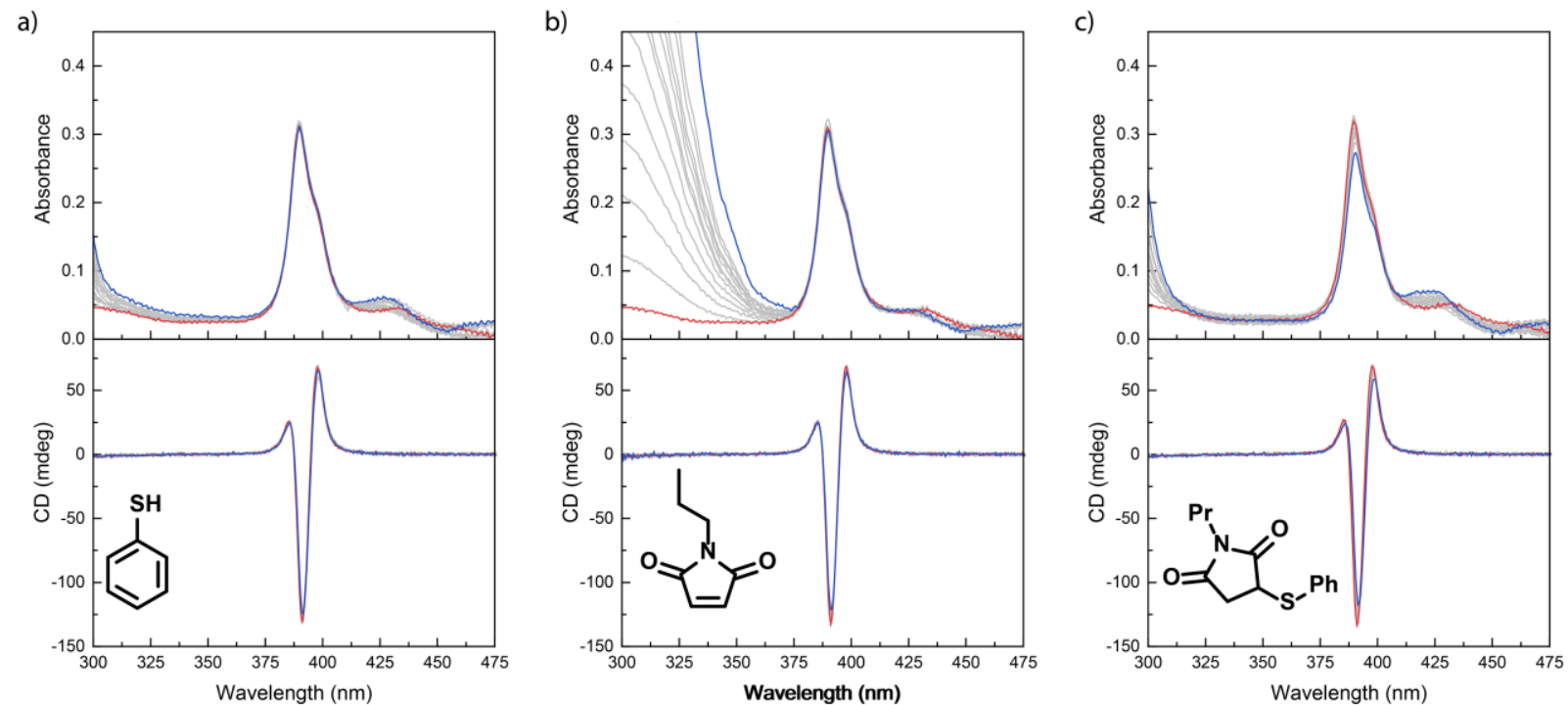

Figure S22 UV-Vis (top panels) and CD (bottom panels) spectra corresponding to the titrations of $10 \mu \mathrm{M}$ Zn-1 with PhSH, NPrMal and $\mathrm{MA}$ in dry $\mathrm{MCH}^{*}$ and in the absence of MePip. From the spectra, the results given in Figure 4 in the main text and Figure S17 in the Supporting Information are obtained. The red spectra indicate the spectra without additive, while the blue spectra indicate the spectra collected at maximum additive concentration.

UV-Vis and CD spectra of the titrations $\mathrm{Zn}-1$ with the PhSH, NPrMal and MA in the presence of $0.5 \mathrm{mM}$ MePip in dry $\mathrm{MCH}^{*}$

a)

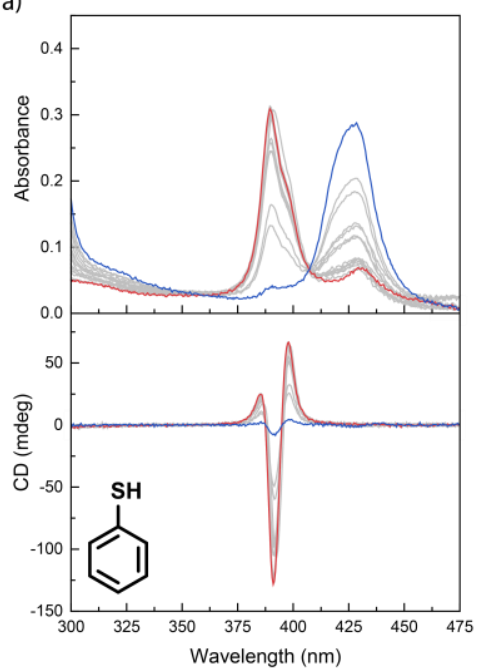

b)

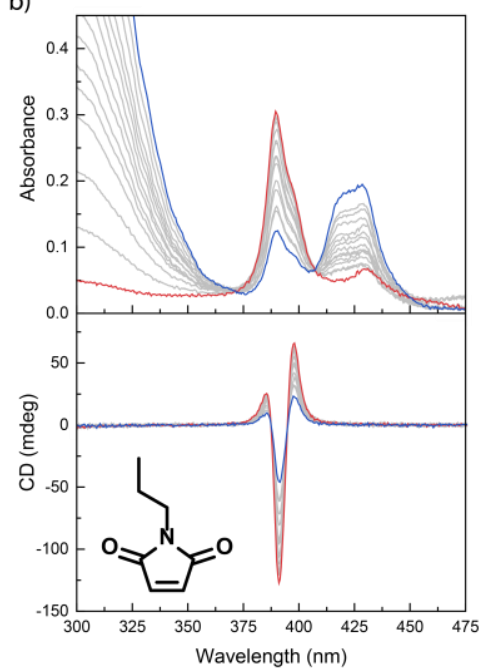

c)

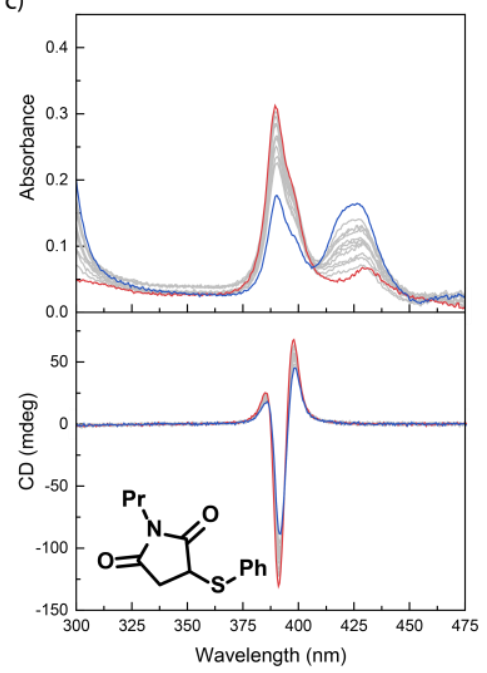

Figure S23 UV-Vis (top panels) and CD (bottom panels) spectra corresponding to the titrations of $10 \mu \mathrm{M}$ Zn-1 with PhSH, NPrMal and $\mathrm{MA}$ in dry $\mathrm{MCH}^{*}$ and in the presence of $0.5 \mathrm{mM}$ MePip. From the spectra, the results given in Figure 4 in the main text and Figure S17 in the Supporting Information are obtained. The red spectra indicate the spectra without additive, while the blue spectra indicate the spectra collected at maximum additive concentration. 
UV-Vis and CD spectra of the titrations $\mathrm{Zn}-1$ with NPrMal in the presence of $5 \mathrm{mM} \mathrm{PhSH}$ in dry $\mathrm{MCH}^{*}$

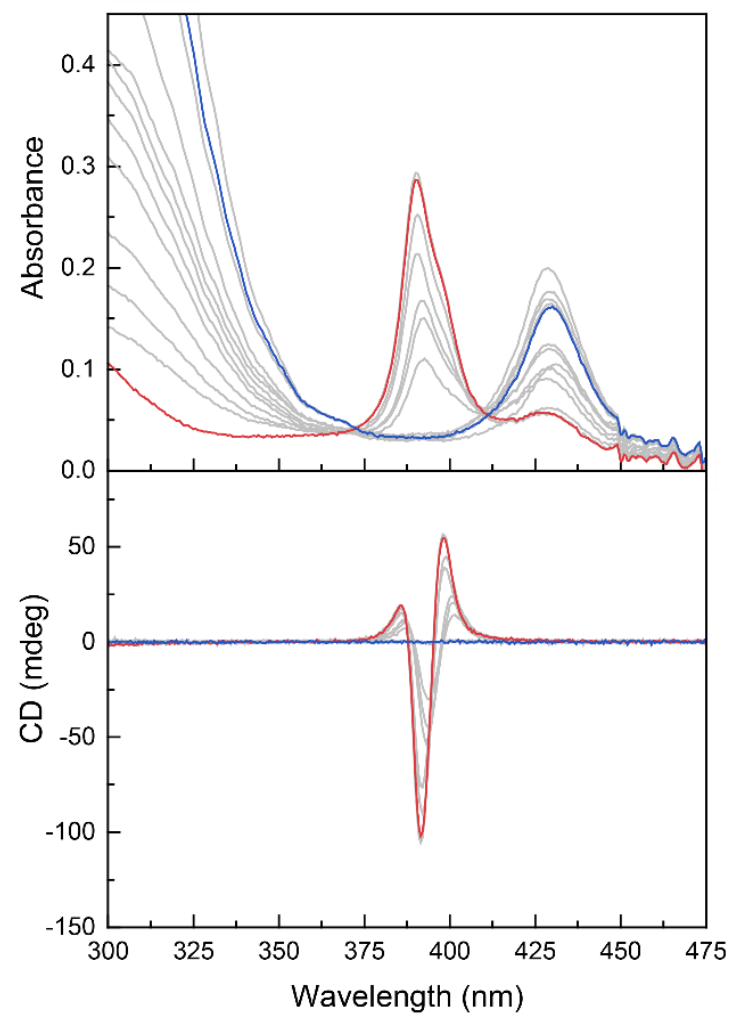

Figure S24 UV-Vis (top panel) and CD (bottom panels) spectra corresponding to the titrations of $10 \mu \mathrm{M}$ Zn-1 with NPrMal in dry $\mathrm{MCH}^{*}$ and in the presence of $5 \mathrm{mM}$ PhSH. From the spectra, the results given in Figure S18 in the Supporting Information are obtained. The red spectra indicate the spectra without additive, while the blue spectra indicate the spectra collected at maximum additive concentration. 
a)

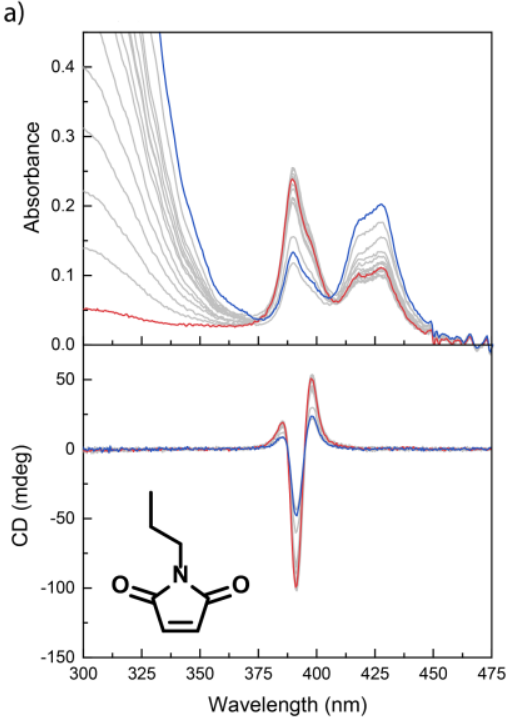

b)

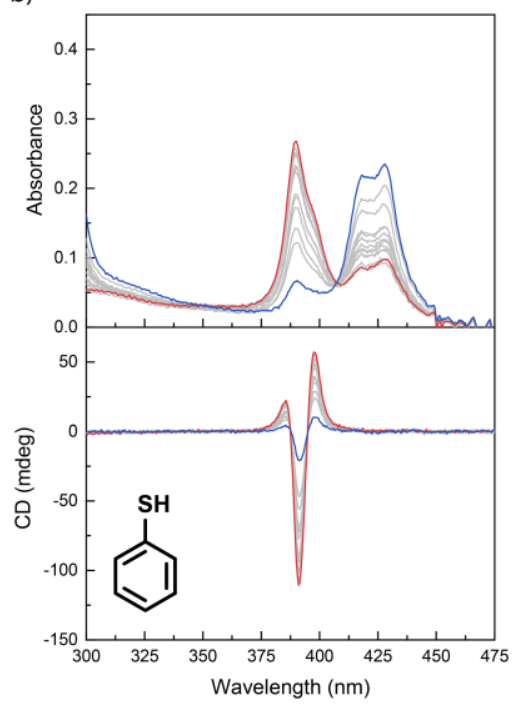

With $0.5 \mathrm{mM}$ Me4Pip

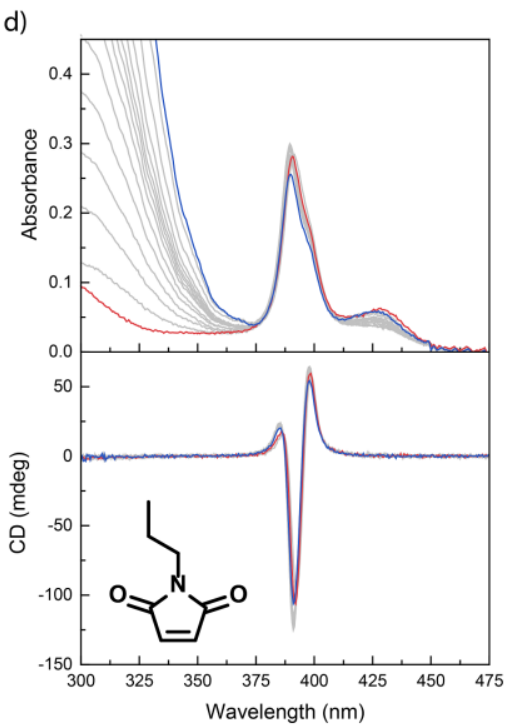

e)

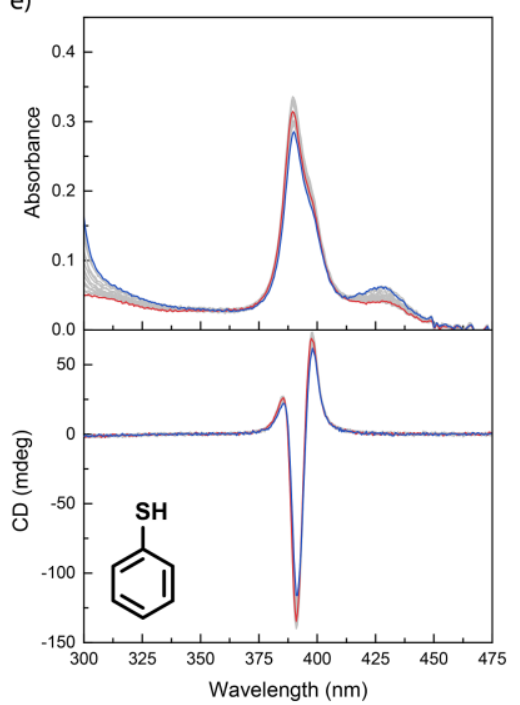

c)

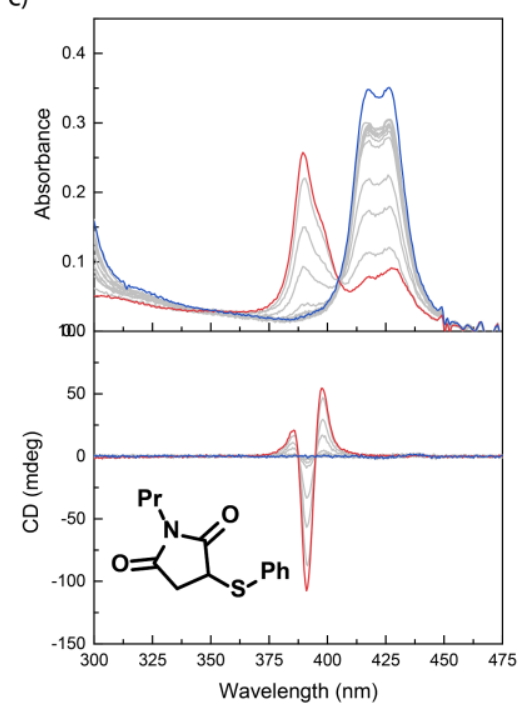

f)

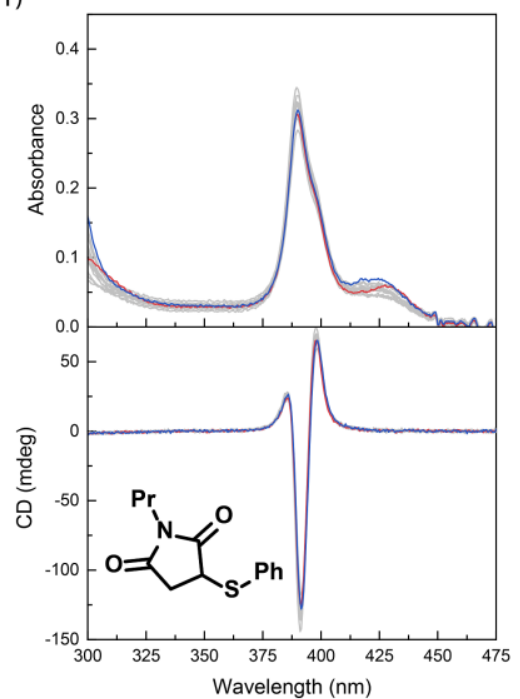

Figure S25 UV-Vis (top panels) and CD (bottom panels) spectra corresponding to the titrations of $10 \mu \mathrm{M}$ Zn-1 with NPrMal (a, d), PhSH (b, e) and MA (c, f) in dry $\mathrm{MCH}^{*}$ and in the presence of $0.5 \mathrm{mM}$ pyridine (top row, a-c) and Me4Pip (bottom row, d-f) From the spectra, the results given in Figure 6 in the main text and Figure S26 in the Supporting Information are obtained. The red spectra indicate the spectra without additive, while the blue spectra indicate the spectra collected at maximum additive concentration. 
Results titration $\mathrm{Zn}-1$ with PhSH and MA

a)

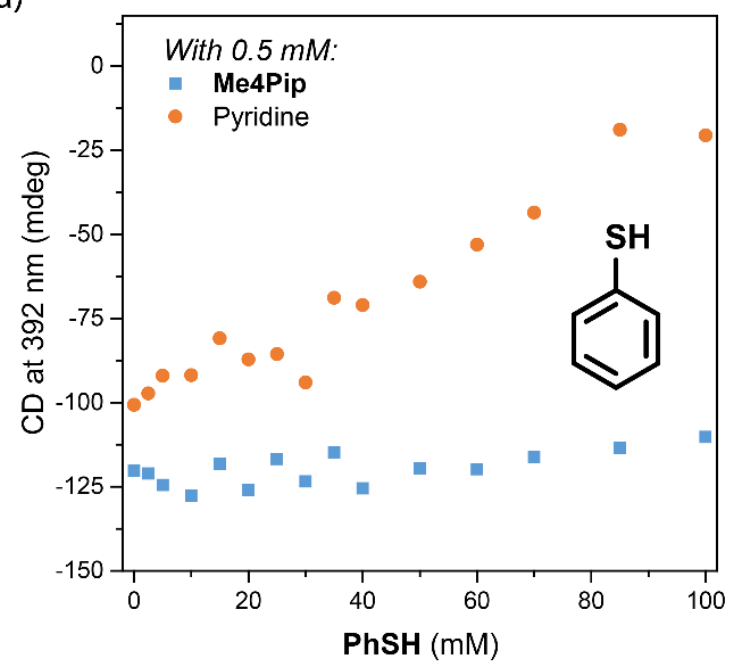

b)

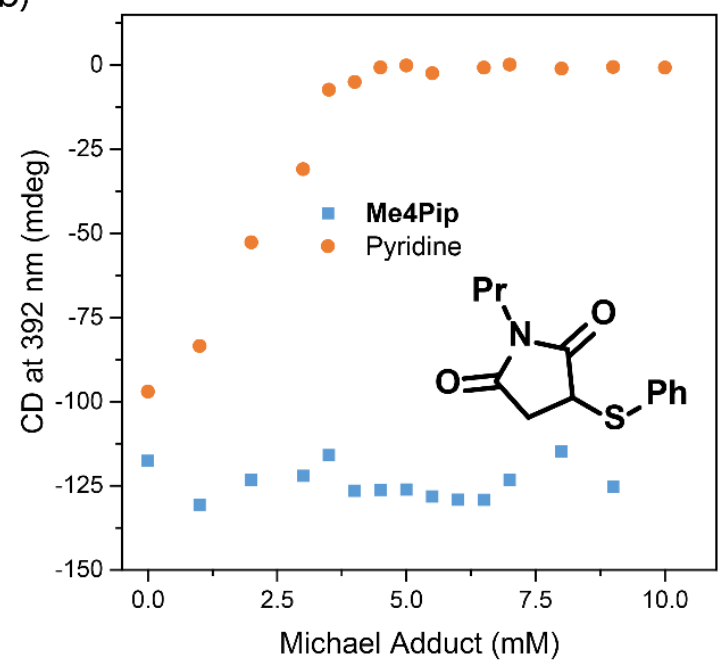

Figure S26 Titrations of $10 \mu$ M solutions of $\mathbf{Z n - 1}$ with PhSH (a) and MA (b) in dry $\mathrm{MCH}^{*}\left(\left[\mathrm{H}_{2} \mathrm{O}\right]\right.$ below detection limit of Karl-Fisher titrator) in the presence of $0.5 \mathrm{mM}$ MePip.

\section{Results titration $\mathrm{H}_{2}-1$ with $\mathrm{MA}$ and $\mathrm{PhSH}$}

a)

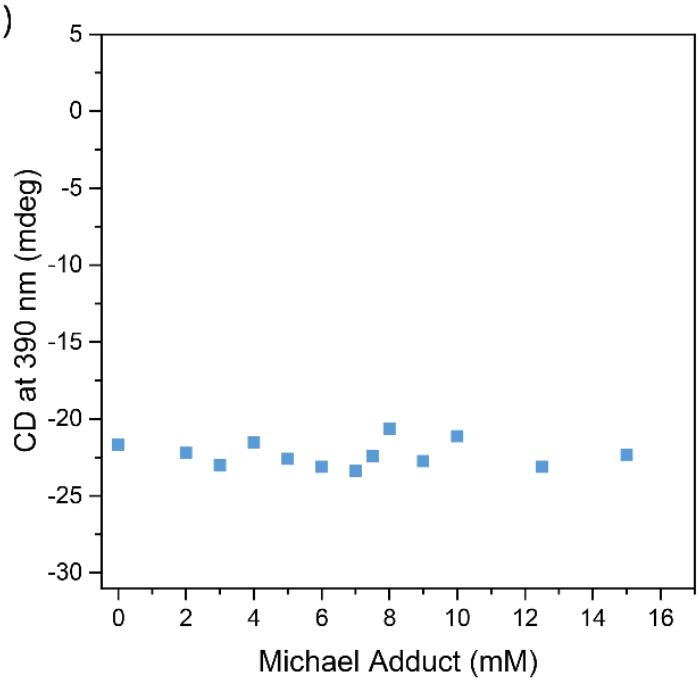

b)

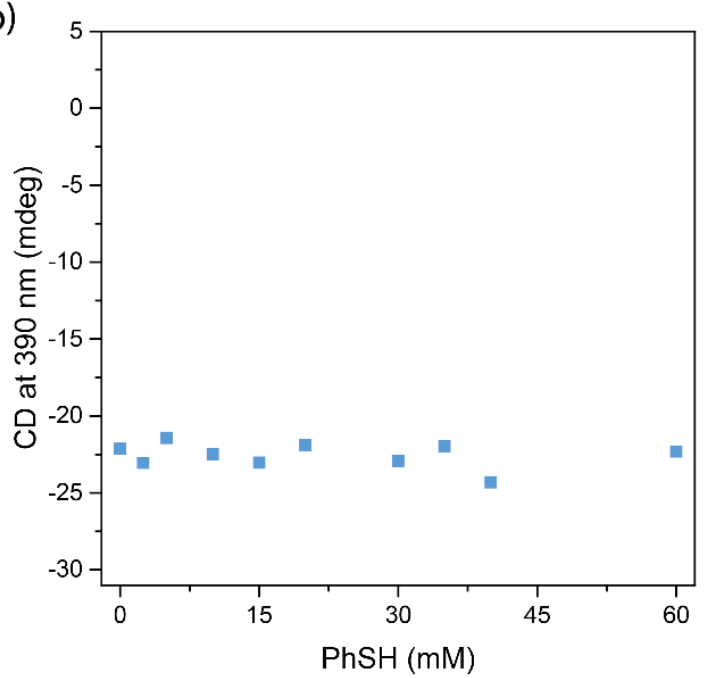

Figure S27 Titrations of $10 \mu \mathrm{M}$ solutions of $\mathbf{H}_{\mathbf{2}}-\mathbf{1}$ with PhSH (a) and $\mathbf{M A}$ (b) in dry $\mathrm{MCH}^{*}$ ( $\left[\mathrm{H}_{2} \mathrm{O}\right]$ below detection limit of Karl-Fisher titrator) in the presence of $0.5 \mathrm{mM}$ MePip. 


\section{UV-Vis and CD spectra titrations $\mathrm{H}_{2}-1$ with NPrMal, $\mathrm{PhSH}$ and MA}
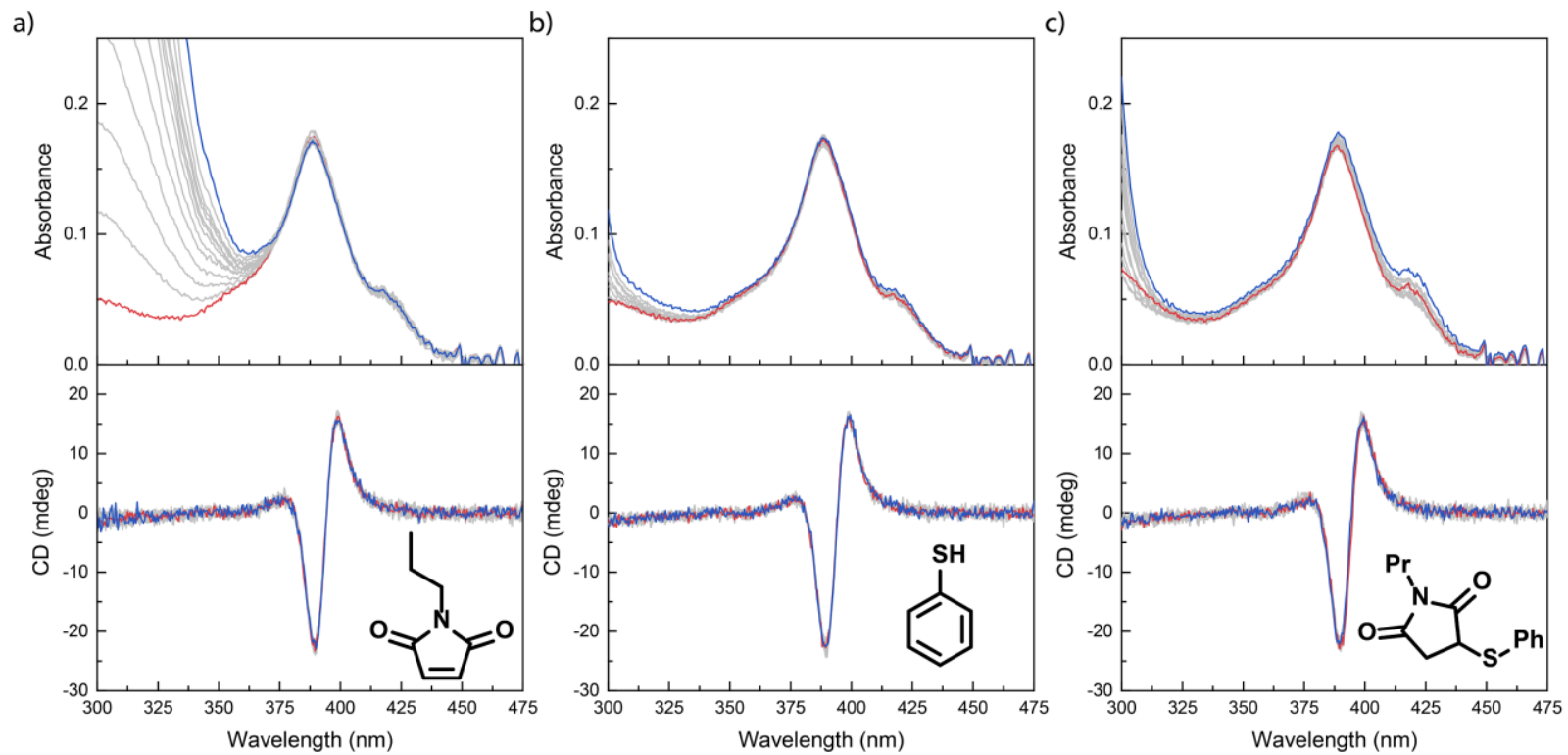

Figure S28 UV-Vis (top panel) and CD (bottom panels) spectra corresponding to the titrations of $10 \mu \mathrm{M}$ solutions of $\mathbf{H}_{\mathbf{2}}-\mathbf{1}$ with NPrMal (a), PhSH (b) and MA (c) in dry $\mathrm{MCH}^{*}\left(\left[\mathrm{H}_{2} \mathrm{O}\right]\right.$ below detection limit of Karl-Fisher titrator) in the presence of $0.5 \mathrm{mM}$ MePip. The spectra are used to obtain the data depicted in Figure $5 b$ in the main text and Figure S27 in the Supplementary Information. The red spectra indicate the spectra without additive, while the blue spectra indicate the spectra collected at maximum additive concentration.

\section{UV-Vis spectra of $\mathrm{H}_{2}-1$ in $\mathrm{MCH}^{*}$ and $\mathrm{CHCl}_{3}$}

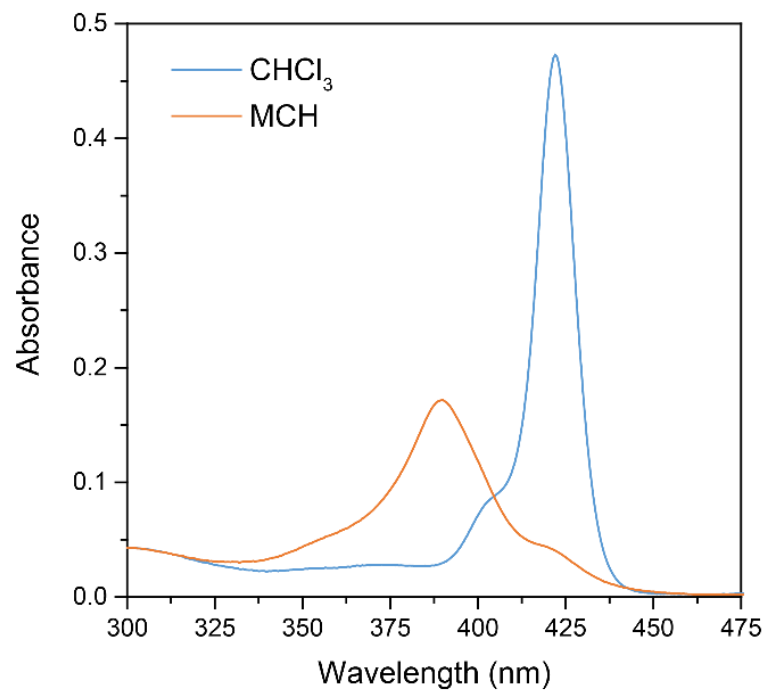

Figure S29 UV-Vis spectra of $10 \mu \mathrm{M}$ solutions of $\mathbf{H}_{\mathbf{2}}-\mathbf{1}$ in ambient $\mathrm{MCH}^{*}$ and $\mathrm{CHCl}_{3}$. 
UV-Vis and CD spectra titrations $\mathrm{Zn}-1$ with $N$-t-butylmaleimide and 4-t-butylthiophenol

a)

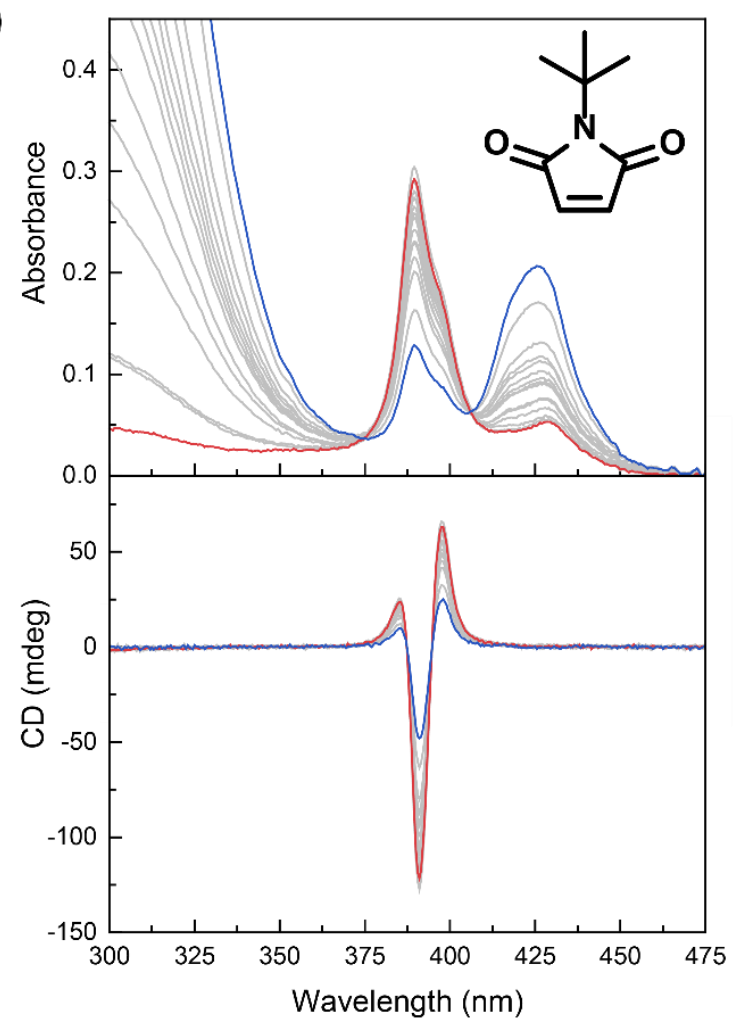

b)

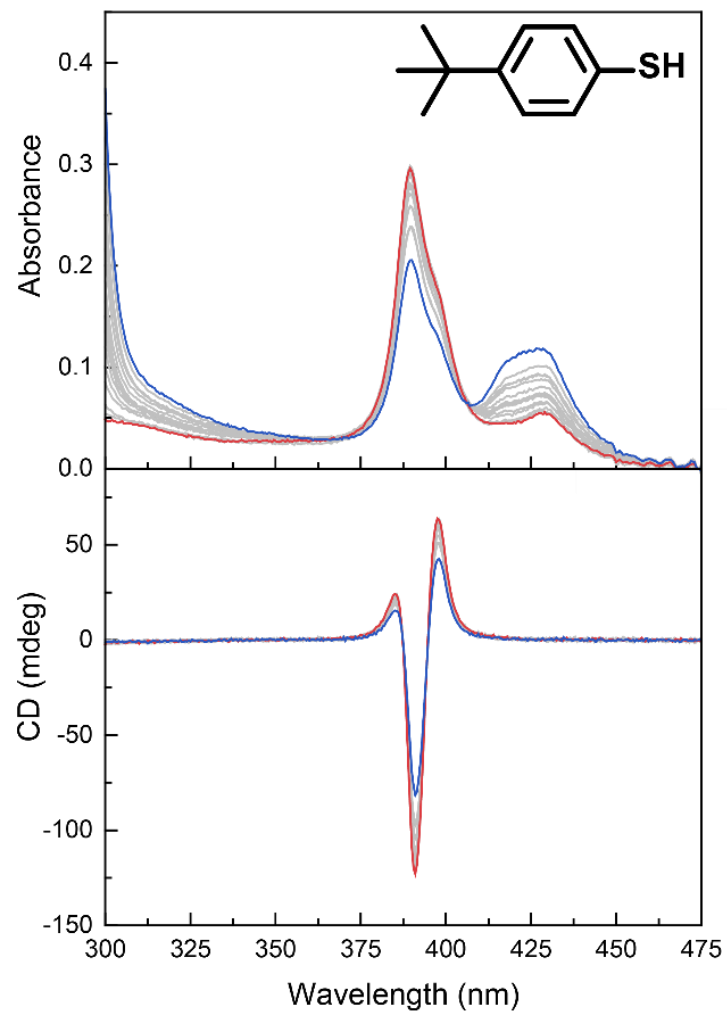

Figure S30 UV-Vis (top panel) and CD (bottom panels) spectra corresponding to the titrations of $10 \mu \mathrm{M}$ solutions of $\mathbf{Z n - 1}$ with tBuMal (a) and tBuPhSH (b) in dry $\mathrm{MCH}^{*}\left(\left[\mathrm{H}_{2} \mathrm{O}\right]\right.$ below detection limit of Karl-Fisher titrator) in the presence of $0.5 \mathrm{mM}$ MePip. The spectra are used to obtain the data depicted in Figure 6 in the main text. The red spectra indicate the spectra without additive, while the blue spectra indicate the spectra collected at maximum additive concentration.

\section{Results titration $\mathrm{Zn}-1$ with bulky substrates}

a)

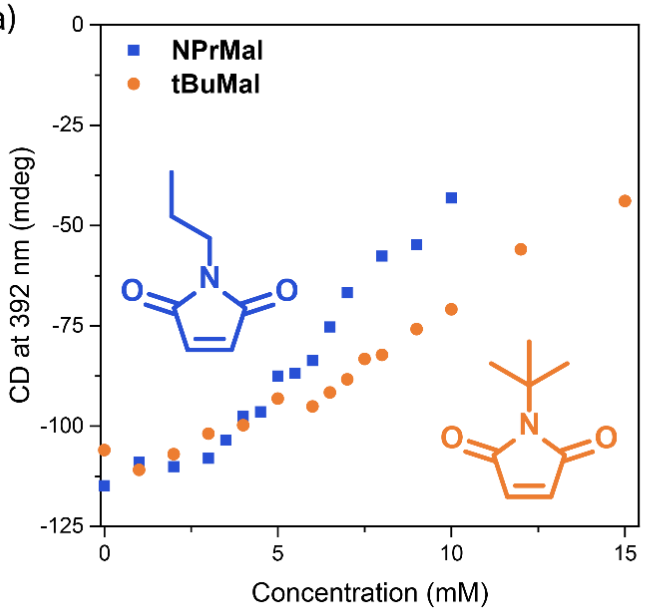

b)

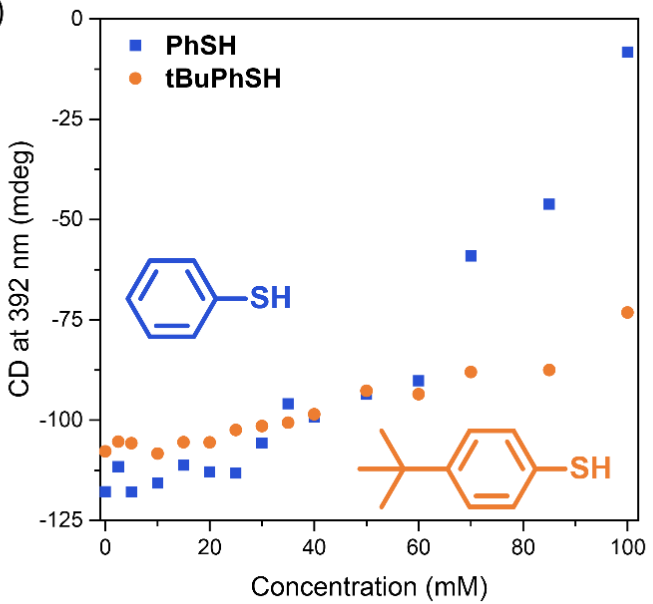

Figure S31 Titrations of $10 \mu \mathrm{M} \mathrm{Zn-1}$ in the presence of $0.5 \mathrm{mM}$ MePip with NPrMal and the bulkier tBuMal (a) and PhSH and tBuPhSH. The titrations were performed in dry $\mathrm{MCH}^{*}$ with [H2O] below the detection limit of the Karl-Fisher titrator. The data for NPrMal and PhSH is taken from Figure 4 in the main text. 


\section{Influence of various amounts of water on the depolymerization by NPrMal and PhSH}

a)

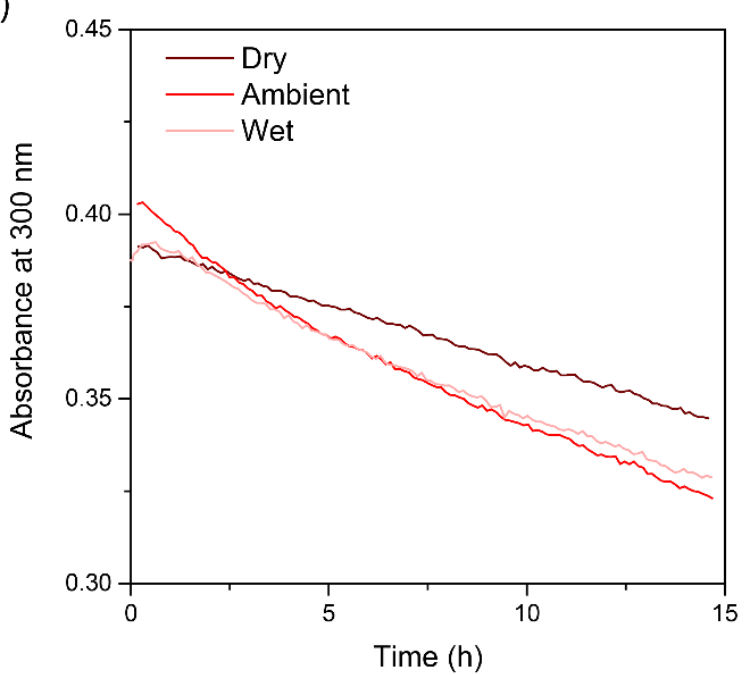

b)

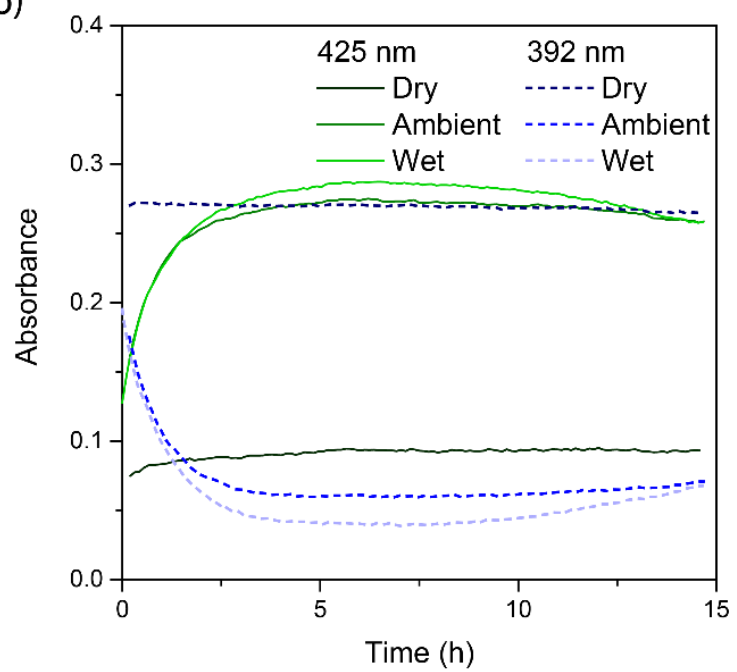

Figure S32 Comparison of the reaction kinetics in $\mathrm{MCH}^{*}$ of the Michael reaction between NPrMal (4 mM) and PhSH (4 mM) which is catalyzed by MePip $(0.5 \mathrm{mM})(\mathrm{a})$ and the stability of supramolecular polymers of $\mathbf{Z n - 1}(10 \mu \mathrm{M})$, indicated by the absorbance of the polymer at $392 \mathrm{~nm}$ (dashed blue lines) and the non-polymerized state (solid green lines) (b). In the dry experiment, the concentration of water was below the detection limit of the Karl Fisher titrator, in the ambient experiment, $\left[\mathrm{H}_{2} \mathrm{O}\right]=12.5 \mathrm{ppm}$ and in the wet experiment, $\left[\mathrm{H}_{2} \mathrm{O}\right]=56.3 \mathrm{ppm}$.

\section{Kinetic profiles of Michael reactions of other substrates}

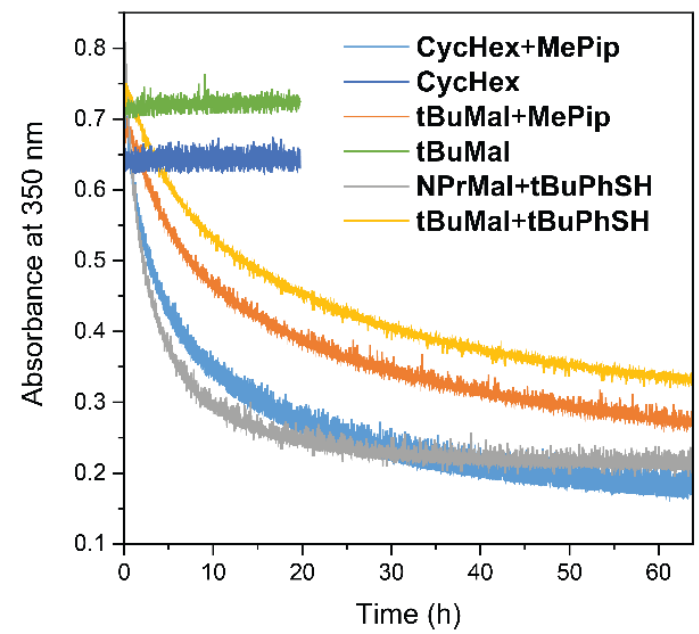

Figure S33 Absorbance at $350 \mathrm{~nm}$ of the reaction mixture of $30 \mathrm{mM}$ CycHex and $30 \mathrm{mM}$ PhSH with and without $0.5 \mathrm{mM}$ MePip (green and dark blue), $10 \mathrm{mM}$ tBuMal and $10 \mathrm{mM}$ PhSH with and without $0.5 \mathrm{mM}$ MePip and $10 \mathrm{mM}$ NPrMal (orange and green) and $10 \mathrm{mM}$ NPrMal with $10 \mathrm{mM}$ tBuPhSH and $0.5 \mathrm{mM}$ MePip (grey) and $10 \mathrm{mM}$ tBuMal with $10 \mathrm{mM}$ tBuPhSH and $0.5 \mathrm{mM}$ MePip (yellow). The reactions were performed in ambient $\mathrm{MCH}^{*}$ with $\left[\mathrm{H}_{2} \mathrm{O}\right]=35.0 \mathrm{ppm}$ and the negative controls were performed in ambient $\mathrm{MCH}^{*}$ with $\left[\mathrm{H}_{2} \mathrm{O}\right]=21.4 \mathrm{ppm}$. 
Time-dependent UV-Vis and CD spectra of $\mathrm{Zn}-1$ in the Michael reaction mixture of PhSH and CycHex
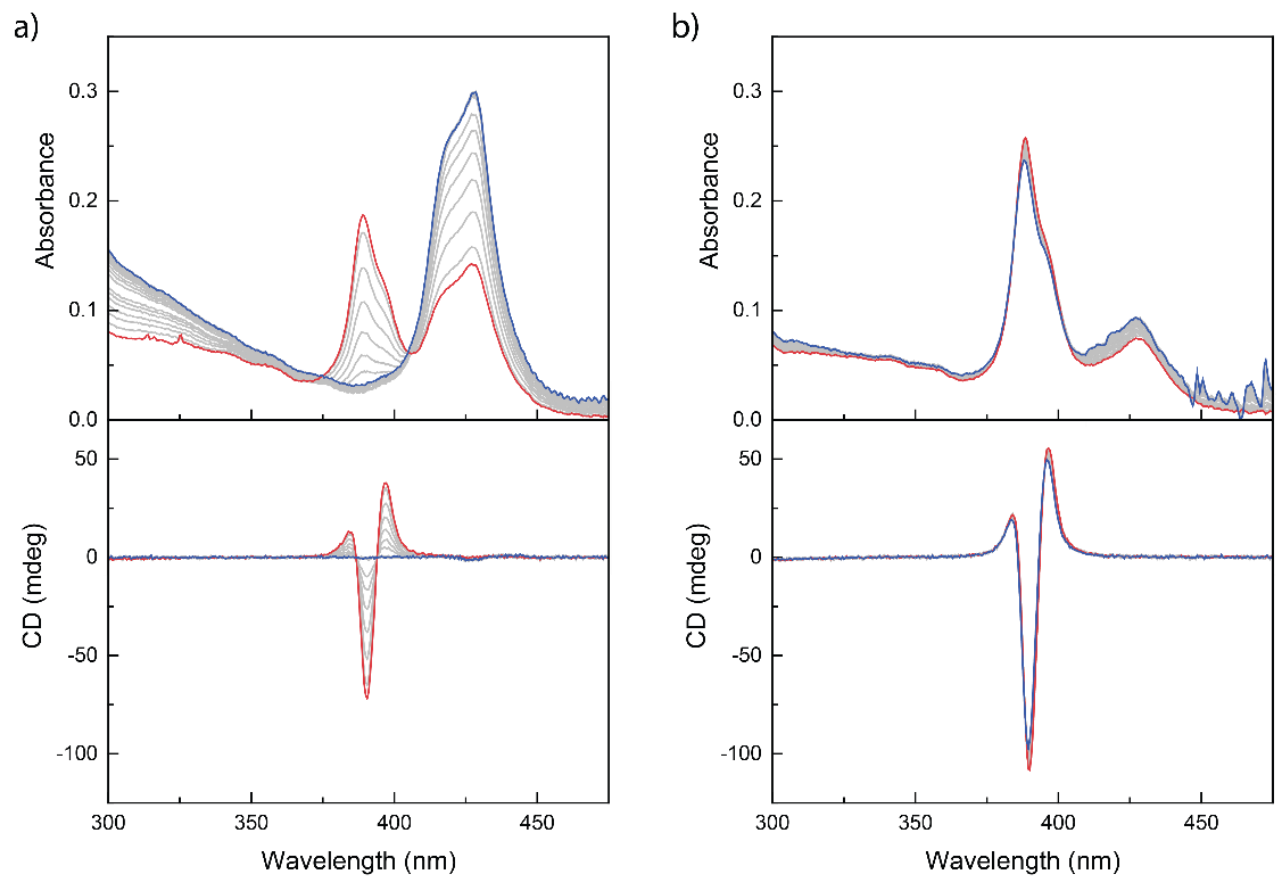

Figure S34 a) Selection of UV-Vis spectra of a solution of $10 \mu \mathrm{M} \mathrm{Zn-1}$ with $0.5 \mathrm{mM} \mathrm{MePip}$ and $10 \mathrm{mM}$ CycHex and PhSH in ambient (a) and dry (b) $\mathrm{MCH}^{*}$ between 0 (red spectrum) and 21 hours (blue spectrum) of the measurement. In the ambient sample, $\left[\mathrm{H}_{2} \mathrm{O}\right]=30.0 \mathrm{ppm}$ and in the dry sample $\left[\mathrm{H}_{2} \mathrm{O}\right]=11.4 \mathrm{ppm}$.

Time dependency absorbance of spectra of $\mathrm{Zn}-1$ in the Michael reaction mixture of $\mathrm{PhSH}$ and CycHex

a)

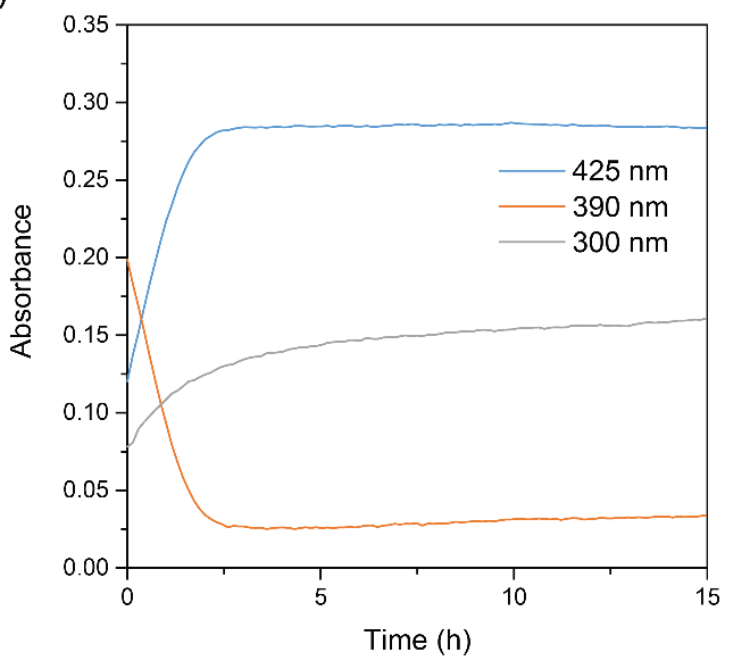

b)

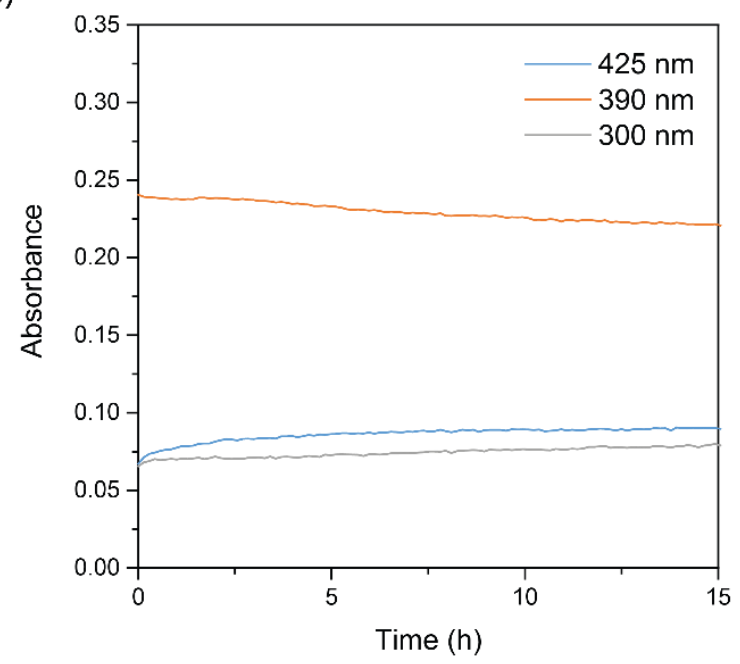

Figure S35 Time dependency of the absorbance at $300 \mathrm{~nm}, 390 \mathrm{~nm}$, and $425 \mathrm{~nm}$ of a solution of $10 \mu \mathrm{M} \mathrm{Zn}-1$ with $0.5 \mathrm{mM}$ MePip and $10 \mathrm{mM} \mathrm{CycHex}$ and $\mathrm{PhSH}$ in ambient (a) and dry (b) $\mathrm{MCH}^{*}$. In the ambient sample, $\left[\mathrm{H}_{2} \mathrm{O}\right]=30.0$ ppm and in the dry sample $\left[\mathrm{H}_{2} \mathrm{O}\right]=11.4 \mathrm{ppm}$. 
Time-dependent UV-Vis and CD spectra of $\mathrm{Zn}-1$ in control experiments of the Michael reaction between PhSH and CycHex

a)

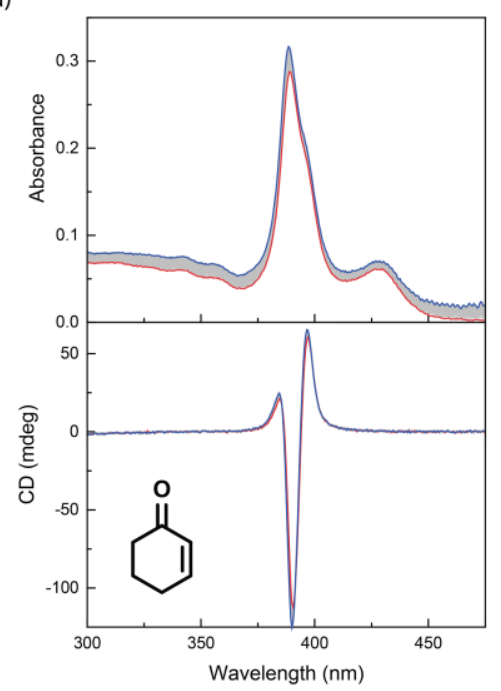

b)

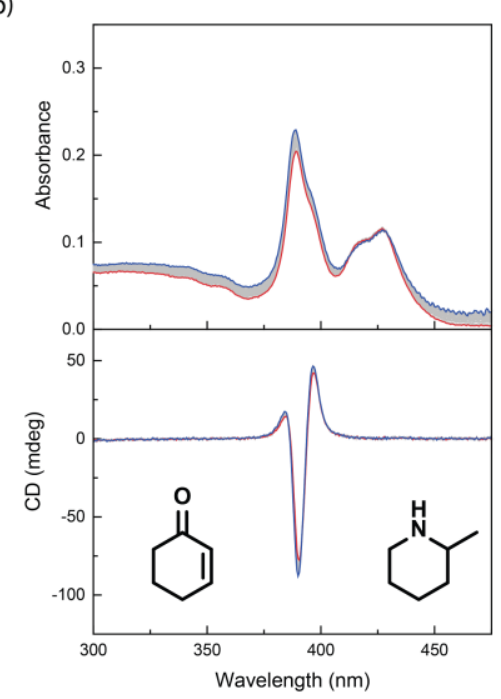

c)

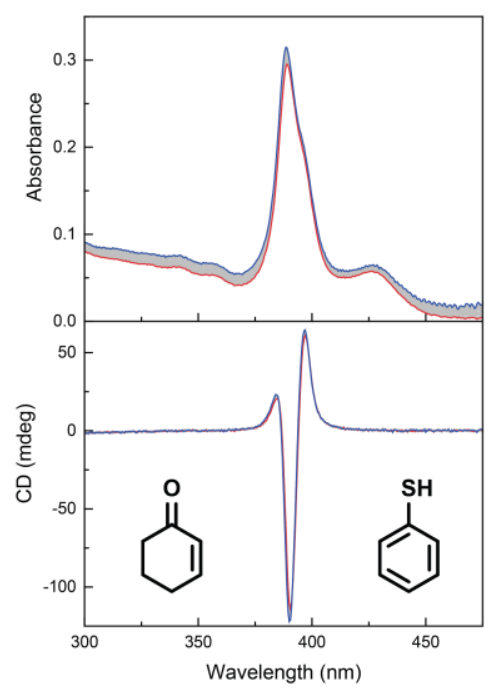

d)

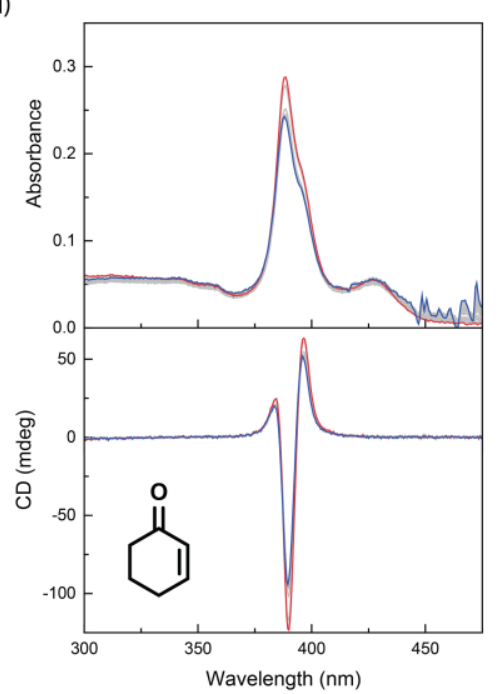

Dry

e)

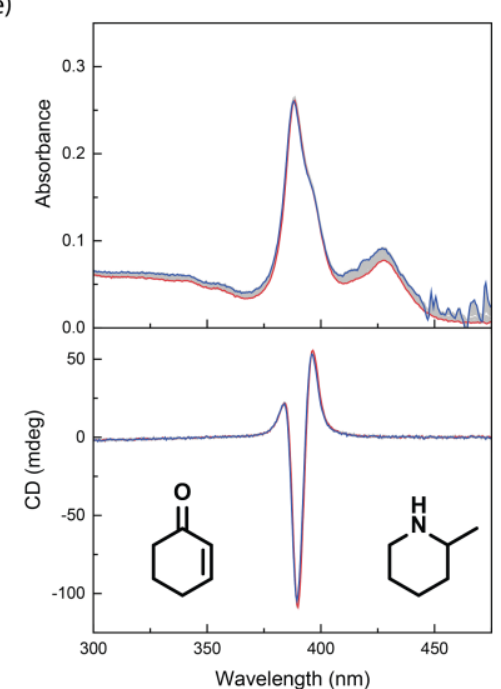

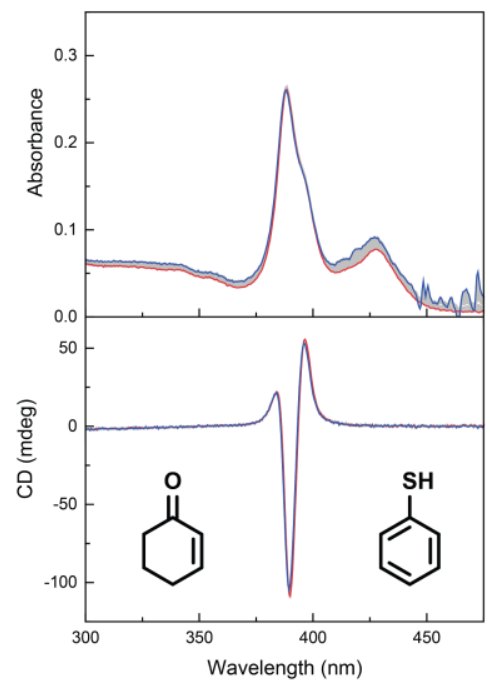

Figure S36 Selection of UV-Vis spectra (top panels) and simultaneously collected CD spectra (bottom panels) of samples of $10 \mu \mathrm{M}$ Zn-1 with $10 \mathrm{mM} \mathrm{CycHex} \mathrm{(a,} \mathrm{d),} 10 \mathrm{mM}$ CycHex with $0.5 \mathrm{mM}$ MePip (b, e) and $10 \mathrm{mM}$ NPrMal with $10 \mathrm{mM}$ PhSH (c, f) in ambient $\mathrm{MCH}^{*}(\mathrm{a}-\mathrm{c})\left(\left[\mathrm{H}_{2} \mathrm{O}\right]=30.0 \mathrm{ppm}\right)$ and dry $\mathrm{MCH}^{*}(\mathrm{~d}-\mathrm{f})\left(\left[\mathrm{H}_{2} \mathrm{O}\right]=11.4 \mathrm{ppm}\right)$. The spectra were collected between 0 hours (red spectrum) and 21 hours (blue spectrum) after sample preparation. 
Time dependency of absorbance of specra of $\mathrm{Zn}-1$ in control experiments of the Michael reaction between PhSH and CycHex

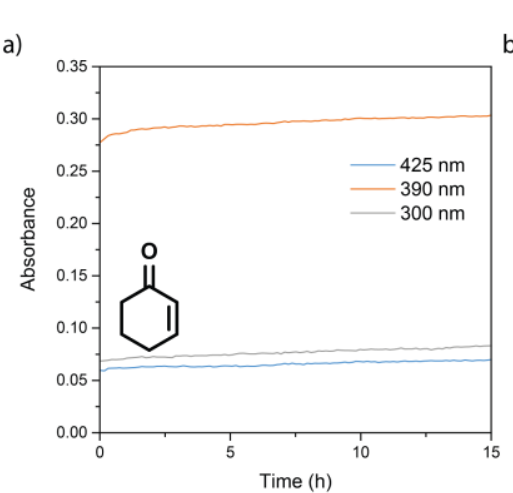

b)

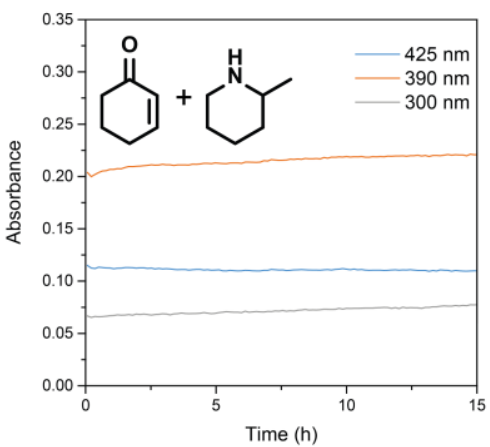

c)

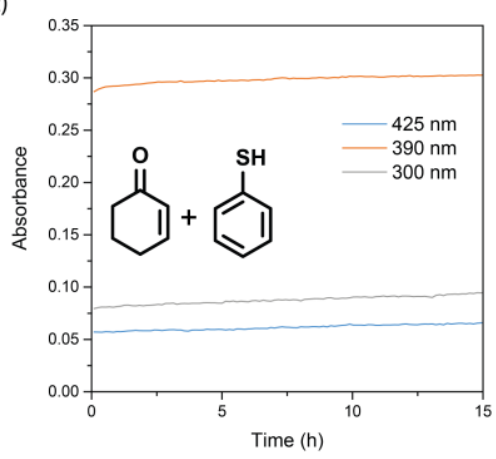

d)

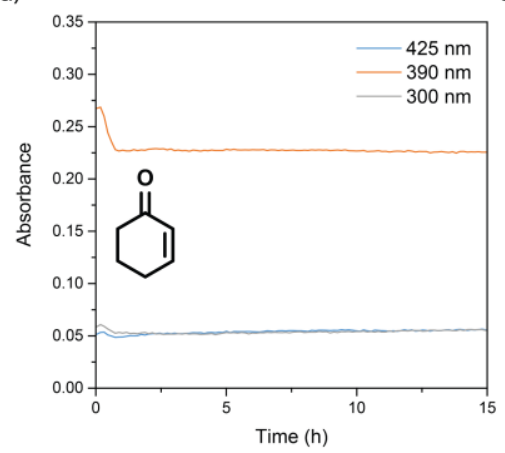

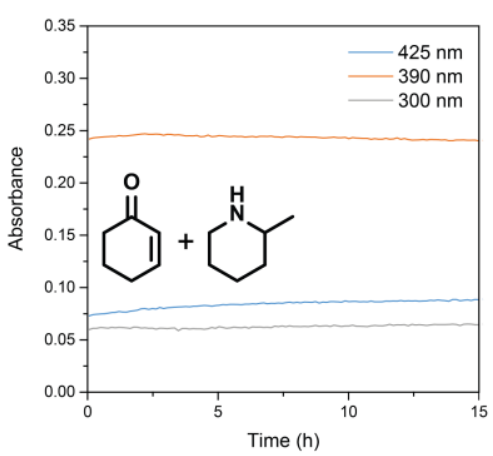

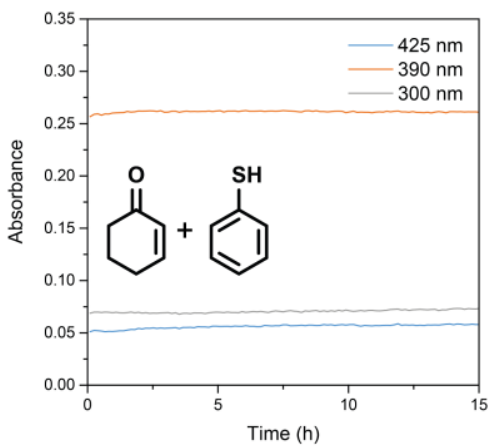

Figure 37 Time dependency of the absorbance at $300 \mathrm{~nm}, 390 \mathrm{~nm}$, and $425 \mathrm{~nm}$ of a solution of $10 \mu \mathrm{M} \mathrm{Zn}-1$ with $0.5 \mathrm{mM}$ MePip and $10 \mathrm{mM}$ CycHex (a, d), $10 \mathrm{mM}$ CycHex with $0.5 \mathrm{mM}$ MePip (b, e) and $10 \mathrm{mM}$ CycHex and $10 \mathrm{mM}$ PhSH (10 mM) in ambient (a-c) and dry (d-f) $\mathrm{MCH}^{*}$. In the ambient sample, $\left[\mathrm{H}_{2} \mathrm{O}\right]=30.0 \mathrm{ppm}$ and in the dry sample $\left[\mathrm{H}_{2} \mathrm{O}\right]=11.4 \mathrm{ppm}$.

\section{Supplementary references}

1. Helmich, F., Lee, C. C., Nieuwenhuizen, M. M. L., Gielen, J. C., Christianen, P. C. M., Larsen, A., Fytas, G., Leclère, P. E. L. G., Schenning, A. P. H. J., Meijer, E. W. Angew. Chem., Int. Ed., 2010, 49, 39393942.

2. Thordarson, P. Chem. Soc. Rev., 2011, 6, 1305-1323.

3. Malkov, A. V., Stončius, S., MacDougall, K. N., Mariani, A., McGeoch, G. D., Kočovský, P. Tetrahedron, 2006, 62, 264-284. 